\title{
Identification of hub genes and biological pathways in hepatocellular carcinoma by integrated bioinformatics analysis
}

\author{
Qian Zhao ${ }^{1}$, Yan Zhang ${ }^{1}$, Shichun Shao ${ }^{2}$, Yeqing Sun ${ }^{\text {Corresp., } 2}{ }^{2}$, Zhengkui Lin ${ }^{\text {Corresp. } 1}$ \\ ${ }^{1}$ College of Information Science and Technology, Dalian Martime University, Dalian, Liaoning, China \\ College of Environmental Science and Engineering, Dalian Martime University, Dalian, Liaoning, China \\ Corresponding Authors: Yeqing Sun, Zhengkui Lin \\ Email address: 554951052@qq.com, 2111897652@qq.com
}

Background: Hepatocellular carcinoma (HCC), the main type of liver cancer in human, is one of the most prevalent and deadly malignancies in the world. The present study aimed to identify hub genes and key biological pathways by integrated bioinformatics analysis.

Methods: A bioinformatics pipeline based on gene co-expression network (GCN) analysis was built to analyze the gene expression profile of HCC. Firstly, differentially expressed genes (DEGs) were identified and a GCN was constructed with Pearson correlation analysis. Then, the gene modules were identified with 3 different community detection algorithms, and the correlation analysis between gene modules and clinical indicators was performed. Moreover, we used the Search Tool for the Retrieval of Interacting Genes (STRING) database to construct a protein protein interaction (PPI) network of the key gene module, and we identified the hub genes using 9 topology analysis algorithms based on this PPI network. Further, we used the Oncomine analysis, survival analysis, GEO data set and random forest algorithm to verify the important roles of hub genes in HCC. Lastly, we explored the methylation changes of hub genes using another GEO data (GSE73003).

Results: Firstly, among the expression profiles, 4130 up-regulated genes and 471 down-regulated genes were identified. Next, the multilevel algorithm which had the highest modularity divided the GCN into 9 gene modules. And, a key gene module (m1) was identified. The biological processes of GO enrichment of $\mathrm{ml}$ mainly included the processes of mitosis and meiosis and the functions of catalytic and exodeoxyribonuclease activity. Besides, these genes were enriched in the cell cycle and mitotic pathway. Furthermore, we identified 11 hub genes, MCM3, TRMT6, AURKA, CDC20, TOP2A, ECT2, TK1, MCM2, FEN1, NCAPD2 and KPNA2 which played key roles in HCC. The results of multiple verification methods indicated that the 11 hub genes had highly diagnostic efficiencies to distinguish tumors from normal tissues. Lastly, the methylation changes of gene CDC20, TOP2A, TK1, FEN1 in HCC samples had statistical significance $(P$-value $<0.05)$.

Conclusion: MCM3, TRMT6, AURKA, CDC20, TOP2A, ECT2, TK1, MCM2, FEN1, NCAPD2 and KPNA2 could be potential biomarkers or therapeutic targets for HCC. Meanwhile, the metabolic pathway, the cell cycle and mitotic pathway might played vital roles in the progression of HCC. 


\section{Introduction}

2 Hepatocellular carcinoma (HCC) is the main type of liver cancer, which causes more than

3700,000 deaths each year (Ni et al. 2020) (Cho et al. 2019). Recently, many studies have

4 demonstrated that multiple genes and cellular pathways participate in the initiation and

5 progression of HCC. The $C D K N 3$ might play an important role in the transformation process

6 from cirrhosis to HCC by analysis of the gene expression omnibus (GEO) data (Jiang et al. 2020).

7 And, $m i R-133 a-3 p$ might inhibit the growth of HCC by analyzing the $m i R-133 a-3 p$ expression

8 and the clinicopathological characteristics of HCC based on GEO data and the Cancer Genome

9 Atlas (TCGA) data (Liang et al. 2018). Besides, DUXAP8 might be involved in the biological

10 processes such as cell cycle, cell division and cell proliferation in $\mathrm{HCC}$, and the down-regulation

11 of $D U X A P 8$ inhibited the proliferation and invasion of $\mathrm{HCC}$ in vitro (Yue et al. 2019). Previous

12 studies focused on the specific genes in the initiation and progression of HCC, however, the

13 precise molecular mechanisms underlying HCC progression was not clear.

14 In the last decade, the high-throughput platforms were used to generate gene expression

15 profiling in HCC. However, sequencing results are often limited and inconsistent owing to the

16 heterogeneity of samples in independent studies, and due to the fact that most studies focus on

17 one cohort. As such, this study sought to analyze a range of available HCC-related gene

18 expression data sets by integrated bioinformatics analysis, with the goal of identifying potential

19 novel hub genes and key gene module for HCC treatment and diagnosis. Currently, the

20 Weighted Gene Co-expression Network Analysis (WGCNA), for analysis of genetic alteration

21 during tumorigenesis, is increasingly valued as promising tools in medical oncology research.

22 Based on WGCNA, we further explored the gene co-expression network (GCN) analysis 
23 algorithm in this study. Such as, multiple machine learning algorithms were used to improve the 24 reliability of the gene modules.

25 Besides, DNA methylation plays a role in genome stability and gene expression (Esteller \& 26 Herman 2002). In particular, aberrant DNA promoter methylation is an important mechanism for 27 loss of gene function in tumors (Ohtani-Fujita et al. 1993) (Jarrard et al. 1998). Given that 28 methylation is now known to play important roles in cancer, it is of great significance to detect 29 DNA methylation of hub genes in HCC in this study.

30 It is vital to classify and detect the key biological pathways and hub genes participated in 31 the initiation and progression of HCC. Firstly, we obtained the transcriptome data set of HCC 32 and normal tissues from TCGA (Hutter \& Zenklusen 2018), and used the FC- $t$ algorithm to 33 identify differentially expressed genes (DEGs). Next, the Pearson correlation analysis was used 34 to construct a GCN, and three community detection algorithms (multi-level, label-propagation, 35 edge-betweenness) were used to identify gene modules. Then, the correlation analysis between 36 gene modules and clinical indicators was performed, and a key gene module was identified.

37 Herein, we performed GO/Reactome enrichment analysis on key gene module to explore the 38 biological significance. Then, the protein protein interaction (PPI) network of the key gene 39 module was constructed using the Search Tool for the Retrieval of Interacting Genes (STRING) 40 database (Szklarczyk et al. 2011), and the hub genes were identified based on PPI network. 41 Moreover, we used the Oncomine analysis, survival analysis, GEO data set and ROC curve to 42 verify the important roles of hub genes in HCC. Finally, we explored the methylation of hub 43 genes using GEO data (GSE73003).

\section{Materials and Methods}


45

46

47

48

49

50

51

52

53

54

55

56

57

58

59

60

61

62

63

64

65

66

67

\subsection{Data collection and preprocessing}

A workflow of this study is shown in Figure 1. The HCC gene expression profiles used in this study were downloaded from TCGA (https://cancergenome.nih.gov/), which was processed using the RNA-sequencing platform, and contained 416 samples, including $367 \mathrm{HCC}$ samples and 49 normal samples. In order to avoid the interference of genes with lower expression on subsequent analysis, the gene whose maximum FPKM value was less than 1 in tumor or normal samples was removed. Then, the outliers from HCC samples were removed by hierarchical clustering with R function hclust() in the stats package (v3.6.1), and euclidean distance was used in the clustering process. In this study, FC-t algorithm (Chen et al. 2018) was used to identify DEGs. The fold change of each gene's FPKM value between cancer and normal tissues was calculated. Next, the differential expression analysis was carried out on the basis of $t$-test using the t.test() in stats R package (v3.6.1). Only genes with the fold change $\geqslant 2$ or fold change $\leqslant$ 0.5 and $P$-value $<0.05$ were regarded as DEGs.

\subsection{Construction of GCN and identification of gene modules}

Pearson correlation analysis was used to construct a correlation matrix between pairwise DEGs with FPKM values in cancer tissues (Chang et al. 2019). And Pearson correlation analysis was implemented using the cor.test() in stats R package (v3.6.1). Then Pearson correlation coefficient $|\mathrm{PCC}| \geq 0.65$ and $P$-value $<0.05$ was set as the cut-off criteria to screen the interaction between two genes. The reserved interactions were represented by networks, the largest of which was the GCN. We clustered all genes (nodes) in the GCN with three different community detection algorithms, including multi-level (Blondel et al. 2008), label-progration (Raghavan et al. 2007) and edge-betweenness (Newman \& Girvan 2004), which were performed by R functions multilevel.community(), label.propagation.community(), edge.betweenness.community() in 
68 igraph package (v1.2.4) (Csardi \& Nepusz 2006). Then the modularity was used to evaluate the

69 clustering results, so as to select the optimal module identification result.

70 2.3. Association analysis between gene modules and clinical indicators

71 Principal component analysis (PCA) (Wold et al. 1987) was used to analyze the gene expression

72 profiles in each module by using the $\operatorname{prcomp}()$ in stats R package (v3.6.1). Then the first

73 principal component was defined as the module eigengenes (MEs). We believed that the four

74 clinical indicators of event, $T, N$, and $M(T$ referred to the primary tumor stage, $N$ referred to the

75 regional lymph node involvement stage and $M$ referred to the distant metastasis stage) were of

76 great significance for judging the initiation and progression of tumors, so an association matrix

77 was constructed based on the correlation analysis between the MEs and event, $T, N$ and $M$, which

78 was calculated by the Pearson correlation analysis. Then, the gene module which was highly

79 related to clinical indicators was selected as the key gene module.

80 To further verify the importance of the key module, Cox proportional hazards regression

81 model was used to perform survival analysis on genes in all modules, and $P$-values of Cox

82 regression results were obtained (the survival analysis results came from the online analysis tool

83 onclnc (http:// www.oncolnc.org/)). Further, the prognostic significance (PS-value, calculated in

$84 \operatorname{logs}$ as $-\lg P$-value) was used to measure the importance of a gene, and the PS-value of a module

85 is the sum of the PS-values of all genes in this module. Obviously, the larger the PS-value of a

86 module, the more important the module is

\section{2.4. GO/Reactome enrichment analysis}

88 For the biological significance of the key gene module, the genes in which were enriched with

89 the biological processes provided by the GO database (http://geneontology.org/) and the

90 signaling pathways provided by the Reactome database (https://reactome.org/). GO enrichment 
91 analysis was implemented with the enrichGO() in clusterProfiler R package (v3.12.0), and

92 Reactome enrichment analysis was performed by logging in the online database, $P$-value $<0.05$

93 was considered statistically significant. Meanwhile, the top 20 GO terms and signaling pathways

94 with the lowest $P$-value were selected for further research. At last, the GO terms were

95 categorized with QucikGO database (https://www.ebi.ac.uk/QuickGO/).

96 2.5. Construction of PPI network and analysis of hub genes

97 The online database STRING (https://string-db.org/) was applied to construct a PPI network of

98 the genes in key gene module and analyze the functional interactions between proteins. A

99 confidence score $\geq 0.400$ was set as significant. Subsequently, the result was visualized using

100 Cytoscape software (v3.7.1) (Shannon et al. 2003), and nine topology analysis algorithms

101 (DNMC, MNC, Degree, EPC, BottleNeck, Closeness, Radiality, Betweenness, Stress) provided

102 by the cytohubba (Chin et al. 2014) plug-in were used to calculate the importance of nodes

103 (genes) in the PPI network. For details of the nine topology analysis algorithms, please refer to

104 the literature Chin et al. 2014. Further, five genes with the highest score in each algorithm were 105 merged together as the hub genes.

\section{2.6. Validation of Hub Genes}

107 Firstly, the mRNA expression of hub genes was explored in common cancer using Oncomine 108 database (https://www.oncomine.org). The parameters were set as follows: THRESHOLD $(P$ $109 \mathrm{VALUE})=0.05$, THRESHOLD $(\mathrm{FOLD}$ CHANGE $)=2$. Then the online analysis tool onclnc was 110 used for the survival analysis of hub genes. And the cancer was set as LIHC, lower percentile 111 was set as 20, and upper percentile was set as 20. Furthermore, we downloaded a test data set, 112 GSE138485, from the GEO (https://www.ncbi.nlm.nih.gov/geo/), and this data set included 64 
113 paired normal and HCC samples (Table S1). The $t$-test was used to verify the differential

114 expression of the hub gene in GSE138485. Moreover, ROC curve and AUC were used to detect

115 the ability of hub genes to distinguish tumors from normal tissues.

116 2.7. DNA methylation analysis

117 The gene methylation profiling data set GSE73003 was downloaded from the GEO (Table S2). It

118 included 40 paired normal and HCC samples from 20 patients. We found the methylation

119 changes of the hub genes in GSE73003, and then used $t$-test to identify the genes whose

120 methylation changed significantly.

\section{Results}

\section{3.1. Identification of DEGs in expression profiles}

123 The HCC gene expression profiles contained 416 samples, including $367 \mathrm{HCC}$ and 49 normal 124 samples, and the original HT-Seq-FPKM data included 60,483 genes in total. The genes with

125 lower expression were removed. Then, the remained 14,129 genes (Table S3) were used for 126 hierarchical clustering to obtain a data set for further analysis. The result showed that there were 127 three outlier samples should be removed, TCGA-DD-AAEB, TCGA-CC-5259 and TCGA-FV128 A4ZP (Figure S1). Using bioinformatics approaches, a total of 4,601 DEGs between HCC and 129 normal samples were identified, including 4,130 up-regulated and 471 down-regulated genes 130 (Figure 2, Table S4).

131 3.2. Construction of GCN and identification of gene modules 
132 Pearson correlation analysis was used to construct a correlation matrix with FPKM values

133 between pairwise DEGs. In total, there were 21,169,201 interactions. After filtered, 2,859 genes

134 and 57,340 interactions were kept and imported into Cytoscape software for visualization. In

135 total, 95 networks were built (Figure 3). The result showed that there were 2,583 genes in the

136 large network, while there were fewer than 20 genes in each smaller one. After removed the

137 small networks, the largest one which referred to GCN were kept for the further research.

138 To obtain more accurate and objective clustering results, three community discovery

139 algorithms were used to cluster all genes (nodes) in the GCN. The modularity of each algorithm

140 was shown in Table 1, and the modularity of multi-level was 0.6009015 , label-propagation was

1410.3748268 and betweenness was 0.4815381 , respectively. The multi-level algorithm clustering

142 result with the highest modularity was performed for subsequent analysis. A total of nine

143 modules were identified after removing the modules with less than 50 genes (Figure 4, Table S5).

144 The network density of these nine modules was shown in Table 2; the density of $\mathrm{m} 9$ was the

145 lowest one, 0.065630124 . It was worth noting that the network density of these nine modules

146 was greater than that of GCN (0.01704045).

\section{3.3. Identification of key gene module and GO/Reactome enrichment analysis}

148 The first principal component of PCA result which performed on the gene expression profiles in

149 each module defined as the MEs (Table S6). Furthermore, an association matrix was constructed

150 based on the correlation analysis between the MEs and the clinical indicators, including event, $T$,

$151 N$ and $M$, which was calculated by the Pearson correlation analysis. In this process, a key gene

152 module $\mathrm{m} 1$ was obtained from the association matrix, and the correlation coefficient between $\mathrm{m} 1$ 153 and $T$ was the maximum $0.259, \mathrm{ml}$ and event was 0.179 , as well as $\mathrm{m} 1$ and $N$ was 0.080 (Figure 
154 5). In addition, we calculated the PS-values of all modules, and the PS-value of module m1 was 155 the largest (Figure 6).

156 To further investigate the function of identified genes in $\mathrm{m} 1$, GO enrichment analysis was 157 performed to analyze functional enrichment (Table S7). The top 20 biological processes

158 enriched in GO terms were shown in Figure 7. The genes in $\mathrm{m} 1$ mainly participated in biological 159 processes associated with the process of mitosis and meiosis, and the functions of catalytic and 160 exodeoxyribonuclease activity. Moreover, the biological processes of key gene module mostly 161 occurred in the chromosomal region. Additionally, the genes in m1 were enriched in many 162 Reactome signaling pathways (Table S8), which mainly included the cell cycle and mitotic 163 (Table 3).

164 3.4. Construction of PPI network and identification of hub genes

165 Based on the STRING database, a PPI network for all genes in the $\mathrm{m} 1$ was constructed (Figure 166 8). Nine topological analysis algorithms provided by cytohubba plug-in were used to calculate 167 the importance of node (gene) in the PPI network (Table S9). The five genes with the highest 168 score in each algorithm were merged together to be the hub genes in this study. Finally, 16 hub 169 genes were identified, NUSAP1, MCM3, TRMT6, RFC3, POLA2, AURKA, CDC20, TOP2A, 170 ECT2, TK1, MCM2, FEN1, NOP58, GINS2, NCAPD2 and KPNA2 (Table 4). It was worth 171 noting that the 16 hub genes were all up-regulated genes.

\section{3.5. Validation of hub genes}

173 Firstly, the mRNA expression of 16 hub genes in liver cancer was explored using Oncomine 174 analysis. The result showed that 13 hub genes were up-regulated in liver cancer as shown in 175 Figure 9. Then we found that the expression levels of 13 hub genes were significantly related 
176 with worse overall survival (OS) (Logrank $P$-value $<0.05)$ (Figure 10). After the merger, a total

177 of 11 genes meet the above two requirements, which included MCM3, TRMT6, AURKA, CDC20, $178 T O P 2 A, E C T 2, T K 1, M C M 2, F E N 1, N C A P D 2$ and KPNA2. And the following focuses on these 17911 hub genes.

180 Further, the data of GEO (GSE138485) showed that the RPKM of 11 hub genes were 181 significantly (all $P$-values $<0.005$ ) up-regulated in HCC samples compared with normal samples 182 (Figure 11). Moreover, based on the RPKM of 11 hub genes in the GEO data set, we used ROC 183 curve and AUC to classify HCC and normal samples. The results showed that the whole 11 hub 184 genes had highly diagnostic efficiencies to distinguish tumors from normal tissues (AUC $>0.87$ 185 and $P$-value $<1.0 \mathrm{E}-06$ ) (Figure 12).

186 3.6. DNA methylation analysis of the hub genes

187 Among the 11 hub genes, we found the methylation of gene CDC20, TOP2A, TK1, FEN1 were 188 significantly changed in the gene methylation profiling data set (GSE73003) $(P$-value $<0.05)$, 189 and they were hypomethylation in the HCC samples (Table 5). It was noted that the expression 190 (RPKM) of CDC20, TOP2A, TK1, FEN1 were significantly higher in HCC samples compared to 191 normal tissues.

\section{Discussions}

193 On the global scale, HCC is a major contributor to both cancer incidence and mortality.

194 Understanding the molecular mechanism of HCC is of critical importance for early detection, 195 diagnosis, and treatment. In our study, the HCC mechanism was analyzed by bioinformatics 196 analysis, including DEGs screening, GCN construction, module analysis, hub gene identification 
197 in the PPI network, validation of the hub genes, and DNA methylation analysis of the hub genes.

198 These findings may help us to understand the molecular mechanism of HCC pathogenesis and 199 identify potential biomarkers for the diagnosis and treatment of HCC.

200 From the result of module identification, we found that the network density of each module 201 was greater than that of GCN. It might imply that compare with the other genes in the GCN, the 202 genes in one module perform the same biological function, which also proved the reliability of 203 the multi-level algorithm.

204 GO enrichment analyses showed that the key gene module were associated with many 205 biological processes. Previous reports showed that the tRNA expressed abnormal (GO:0006409, 206 GO:00071431, GO:0051031 and GO:0006403) had a dual role for the promotion and 207 suppression in cancer development (Nientiedt et al. 2016). The tRNA might be involved in cell 208 proliferation process, cell cycle and gene regulation process in cancer (Balatti et al. 2017) 209 (Goodarzi et al. 2015). In tumor cells, the variation of cell function was often influenced by the 210 structure of DNA strand and the conformation of chromosome (GO:0009987 and GO:0071103). 211 DNA was regulated by different functional regions in the nucleus due to its local strand structure 212 abnormality, compression or long-range proximity (Phillippa et al. 2016). Besides, signal 213 transduction by p53 class mediator (GO:0072331) and response to heat (GO:0009408) belonged 214 to response to stimulus (GO:0050896). P53 is a key tumor suppressor (Vogelstein et al. 2000). 215 As a transcription factor, p53 transcribes its target genes to regulate various cellular biological 216 processes, including cell cycle arrest, apoptosis, senescence, energy metabolism, and anti217 oxidant defense, to prevent tumorigenesis (Feng \& Levine 2010). P53 is an important tumor 218 suppressor gene, 30 to $60 \%$ of HCC patients with mutated p53 gene (Hussain et al. 2007). Many 219 GO Terms belonged to cellular process (GO:0009987), DNA replication (GO:00006260) and 
220 protein sumoylation (GO:0016925). The post-translational modification sumoylation is a major

221 regulator of protein function that plays an important role in a wide range of cellular processes

222 (GO:0009987 and GO:0016925) (Wilkinson \& Henley 2010). Furthermore, GO enrichment

223 analyses results showed that the small networks were associated with the detoxification of

224 inorganic compound (GO:0061687), nucleosome assembly and organization (GO:0006334,

225 GO:0034728), metabolism, such as fatty, hormone and neurotransmitter. Similarly, the small

226 gene modules were associated with antigen processing and presentation (GO:0019882),

227 complement activation lectin pathway (GO:0001867), RNA splicing (GO:0008380), negative

228 regulation of lymphocyte (GO:0050672) and centromeric sister chromtid (GO:0070601).

229 Beyond these, previous reports revealed the evidence of meiosis (GO:0140014 and GO:1901990)

230 might be responsible for the proliferation of the tumor cells, such as meiosis error invoked the

231 malignant transformation of germ cell tumor (Ichikawa et al. 2013). Besides, the

232 studies demonstrated that oocyte meiosis might induce the proliferation of the tumor cells ( $\mathrm{Li}$ et

233 al. 2014). It has been reported that increased expression genes which were associated with cell

234 cycle and oocyte meiosis, were associated with the development and progression of HCC (Fujii

235 et al. 2006) (Zhang et al. 2019).

236 According to the validation, the 11 hub genes were good biomarkers in HCC and functioned

237 as tumor promoter. MCM3 complex required for cell cycle regulation of DNA replication in

238 vertebrate cells (Madine et al. 1995). MCM3 were significantly up-regulated in invasive ductal

239 carcinoma (Zhao et al. 2020). Consistent with the findings reported in previous studies (Zhuang

240 et al. 2018) (Yang et al. 2019), our results showed that higher MCM3 expression levels are

241 associated with worse clinical outcomes and a shorter survival times of patients with HCC,

242 thereby highlighting the potential use of $M C M 3$ as a prognostic biomarker. $M C M 2$ is a 
243 promising marker for premalignant lesions of the lung (Yan et al. 1993) (Musahl et al. 1998).

244 Besides, high MCM2 expression has been associated with poor prognosis in HCC patients (Liu et

245 al. 2018) (Li et al. 2019). Previous report showed that the up-regulation of Epithelial cell

246 transforming sequence 2 (ECT2) was significantly associated with early recurrent $\mathrm{HCC}$ disease

247 and poor survival. ECT2 was closely associated with the activation of the Rho/ERK signaling

248 axis to promote early $\mathrm{HCC}$ recurrence. Moreover, knockdown of ECT2 markedly suppressed

249 Rho GTPases activities, enhanced apoptosis, attenuated oncogenicity and reduced the metastatic

250 ability of HCC cells (Chen et al. 2015). The NCAPD2 is a novel candidate genes in ovarian

251 cancer (Tatsumoto \& T. 1999) (Fields \& Justilien 2009). In fact, elevated AURKA expression

252 was observed in several human cancers, such as pancreatic cancer, endometrioid ovarian

253 carcinoma and colorectal cancer liver metastasis, and was associated with poor prognosis

254 (Furukawa et al. 2006). Besides, AURKA promoted cancer metastasis by regulating epithelial-

255 mesenchymal transition and cancer stem cell properties in HCC (Chen et al. 2017). Emerging

256 evidence suggests that KPNA2 plays a crucial role in oncogenesis and early recurrence. KPNA2

257 expression levels were found to be markedly higher in tumor tissue $(83.33 \%, 25 / 30)$ (Feng et al.

258 2014). Besides, nuclear KPNA2 expression was significantly up-regulated $(30.3 \%, 67 / 221)$ in

259 HCC tissues; however, no nuclear expression of KPNA2 in non-tumorous tissues was observed

260 by immunohistochemical assays (Jiang et al. 2014). Both in vitro and in vivo experiments

261 demonstrated that knockdown of KPNA2 reduced migration and proliferation capacities of HCC

262 cells, while over-expression of KPNA2 increased these malignant characteristics (Xinggang et al.

263 2019). TOP2A encodes a $170 \mathrm{kDa}$ nuclear enzyme that controls DNA topological structure,

264 chromosome segregation, and cell cycle progression (Isaacs et al. 1998). TOP2A over-

265 expression, that is, increased level of TOP $2 A$ mRNA and protein, has been detected in HCC 
266 (Panvichian et al. 2015) (Wong et al. 2009). TRMT6, which catalyzes the installation of $\mathrm{m} 1 \mathrm{~A}$ at 267 position 58 of tRNA, is an oncogene in $\mathrm{HCC}$ ( $\mathrm{Li}$ et al. 2017). Clinical significance of TRMT6 in 268 HCC and colon cancers is very important (Wang et al. 2019). TRMT6 knockout HCC cells 269 displayed compromised stemness properties, as reflected by impaired sphere formation and 270 tumor initiating ability, and increased sensitivity to molecular target drug sorafenib (Chen 2019). 271 TRMT6 was up-regulated in HCC tissues, and higher TRMT6 expression levels was correlated 272 with reduced OS $(\mathrm{P}=0.0224)$ and $\mathrm{RFS}(\mathrm{P}=0.0146)$ in patients with primary HCC (Wang et al. 273 2019). The above results indicated that TRMT6 might be a promising prognostic biomarker for 274 poor clinical outcomes in primary HCC patients.

275 Previous results showed that the levels of serum TK1 (thymidine kinase 1) in the primary 276 hepatic carcinoma group were significantly higher than those in the control group and the benign 277 group ( $\mathrm{P}<=0.05)$ (Shen-Jie \& Li 2018) (Zhang et al. 2015). Cell division cycle 20 (CDC20) 278 encodes a regulatory protein interacting with the anaphase-promoting complex/cyclosome in the 279 cell cycle and plays important roles in tumorigenesis and progression of multiple tumors (Liu et 280 al. 2015). Immunohistochemistry result showed that, in the 132 matched HCC tissues, high 281 expression levels of $C D C 20$ were detected in $68.18 \%$ HCC samples, and over-expression of $282 C D C 20$ was positively correlated with gender $(\mathrm{P}=0.013)$, tumor differentiation $(\mathrm{P}=0.000)$, TNM 283 stage ( $\mathrm{P}=0.012), \mathrm{P} 53$ and Ki-67 expression ( $\mathrm{P}=0.023$ and $\mathrm{P}=0.007$, respectively) (Li et al. 2014). 284 The Flap endonuclease (FENI) expression levels were also positively correlated with tumor size $285(\mathrm{P}=0.047<0.05)$, distant metastasis $(\mathrm{P}=0.013<0.05)$ and vascular invasion $(\mathrm{P}=0.024<0.05)$ 286 in HCC ( $\mathrm{Li}$ et al. 2019). Combined with the study in this paper, it was reasonable to speculate 287 that these 11 hub genes might be biomarkers for HCC. 
289 expression states and maintains genome integrity by collaborating with proteins that modify 290 nucleosomes (Jaenisch \& Bird 2003) (Zhong et al. 2016). Altered DNA methylation such as 291 tumor suppressor gene hypermethylation or oncogene hypomethylation is thought to promote 292 tumorigenesis (Ehrlich 2019). Previous reports found that genes including P15, P16, RASSF1A 293 and Retinoblastoma 1 were inactivated in HCC due to promoter hypermethylation of these genes 294 (Fan et al. 2018). In the present study, we identified four highly-expressed hub genes with 295 hypomethylation, $C D C 20, T O P 2 A, T K 1, F E N 1$. Therefore, we might provide more effective 296 diagnostic strategies by these novel biomarkers of HCC.

In accordance with our findings, previous studies have also identified hub genes that 298 participate in HCC (Hua et al. 2020) (Song et al. 2020). Shengni et al. constructed a PPI network based on 176 DEGs. Then four gene modules were identified with Cytoscape MCODE plug-in 300 and 12 hub genes were obtained with integrated survival and methylation analysis. Finally, three genes, KPNA2, TARBP1 and RNASEH2A, were identified as diagnostic and prognostic markers 302 for HCC. However, 176 genes were a little too small to construct a network and identify gene modules, and all of DEGs were up-regulated. It would be better if this article had an association 304 analysis between gene modules and clinical indicators. Another study identified key gene modules with WGCNA. And 29 hub genes were identified based on PPI network. Finally, DAO, PCK2, and HAO1 were determined as prognostic targets for HCC. It was worth noting that the study used Pearson correlation analysis to associate gene modules with clinical indicators, which was classic but a little simple. Besides, they used the "Degree" algorithm to identify hub genes in 309 the PPI network, rather than make full use of the topology of the PPI network. In our study, data analysis was conducted using the integrated bioinformatics analysis based on GCN analysis, which is highly suitable for the analysis of gene expression data. First of all, 4,601 DEGs 
312 (including 4,130 up-regulated and 471 down-regulated genes) were used to construct GCN. In

313 particular, we used three community detection algorithms to identify gene modules, and used

314 modularity to select the optimal module identification result. In addition, Pearson correlation

315 analysis and survival analysis were used to identify key gene module, so the key gene module of

316 HCC in this paper was more accurate, and GO enrichment analysis results also proved the

317 reliability of this module. Lastly, nine topology analysis algorithms were used to identify hub

318 genes in PPI network. And we used the Oncomine analysis, survival analysis, GEO data set and

319 ROC curve to verify the important roles of hub genes in HCC. Therefore, the hub genes

320 identified in the present study are more reliable and comprehensive.

\section{Conclusions}

322 In summary, integrated bioinformatics analysis based on GCN analysis was built to analyze the

323 gene expression profile of HCC, and the hub genes and biological pathways in HCC were

324 identified in this study. MCM3, TRMT6, AURKA, CDC20, TOP2A, ECT2, TK1, MCM2, FEN1,

325 NCAPD2 and KPNA2 could be potential biomarkers or therapeutic targets for HCC. Meanwhile,

326 the metabolic pathway, the cell cycle and mitotic pathway might played vital roles in the

327 progression of HCC. However, we need more experiments to investigate these novel, key and

328 hub genes. Based on these results, the underlying molecular mechanisms of HCC were explored

329 on genetic and molecular levels, which provided new insights into HCC diagnosis and treatment.

330

331 References 
332

333

334

335

336

337

338

339

340

341

342

343

344

345

346

347

348

349

350

351

352

353

354

355

356

357

358

359

360

361

362

363

364

365

366

Balatti V, Nigita G, Veneziano D, Drusco A, Stein GS, Messier TL, Farina NH, Lian JB, Tomasello L, and Liu CG. 2017. tsRNA signatures in cancer. Proceedings of the National Academy of Sciences of the United States of America:201706908. 10.1073/pnas.1706908114

Blondel VD, Guillaume JL, Lambiotte R, and Lefebvre E. 2008. Fast unfolding of communities in large networks. Journal of Statistical Mechanics 2008:0-0. 10.1088/1742-5468/2008/10/P10008

Chang YM, Lin HH, Liu WY, Yu CP, Chen HJ, Wartini PP, Kao YY, Wu YH, Lin JJ, Lu MJ, Tu SL, Wu SH, Shiu SH, Ku MSB, and Li WH. 2019. Comparative transcriptomics method to infer gene coexpression networks and its applications to maize and rice leaf transcriptomes. Proceedings of the National Academy of Sciences of the United States of America 116:3091-3099. 10.1073/pnas. 1817621116

Chen C, Song G, Xiang J, Zhang H, Zhao S, and Zhan Y. 2017. AURKA promotes cancer metastasis by regulating epithelial-mesenchymal transition and cancer stem cell properties in hepatocellular carcinoma. Biochem Biophys Res Commun 486:514-520. 10.1016/j.bbrc.2017.03.075

Chen J, Wang X, Hu B, He Y, Qian X, and Wang W. 2018. Candidate genes in gastric cancer identified by constructing a weighted gene co-expression network. PeerJ 6:e4692. 10.7717/peerj.4692

Chen J, Xia H, Zhang X, Karthik S, Pratap SV, Ooi LL, Hong W, and Hui KM. 2015. ECT2 regulates the Rho/ERK signalling axis to promote early recurrence in human hepatocellular carcinoma. Journal of Hepatology 62:1287-1295. 10.1016/j.jhep.2015.01.014

Chen M. 2019. Dysregulations and functions of RNA modifying enzymes METTL3 and TRMT6 in hepatocellular carcinoma.

Chin CH, Chen SH, Wu HH, Ho CW, Ko MT, and Lin CY. 2014. cytoHubba: identifying hub objects and sub-networks from complex interactome. BMC Systems Biology 8 Suppl 4:S11. 10.1186/17520509-8-s4-s11

Cho K, Ro SW, Seo SH, Jeon Y, Moon H, Kim DY, and Kim SU. 2019. Genetically Engineered Mouse Models for Liver Cancer. Cancers 12. 10.3390/cancers12010014

Csardi G, and Nepusz T. 2006. The igraph software package for complex network research. InterJournal, complex systems 1695:1-9.

Ehrlich M. 2019. DNA hypermethylation in disease: mechanisms and clinical relevance. Epigenetics 14:1141-1163. 10.1080/15592294.2019.1638701

Esteller M, and Herman JG. 2002. Cancer as an epigenetic disease: DNA methylation and chromatin alterations in human tumours. Journal of Pathology 196:1-7. 10.1002/path.1024

Fan G, Tu Y, Chen C, Sun H, Wan C, and Cai X. 2018. DNA methylation biomarkers for hepatocellular carcinoma. Cancer Cell International 18:140. 10.1186/s12935-018-0629-5

Feng L, Zeyan P, Fangjiu L, Rong W, and Yanli L. 2014. Expression of KPNA2 in hepatocellular carcinoma. International Journal of Laboratory Medicine.

Peer) reviewing PDF | (2020:06:49658:3:0:NEW 24 Nov 2020) 
367

368

369

370

371

372

373

374

375

376

377

378

379

380

381

382

383

384

385

386

387

388

389

390

391

392

393

394

395

396

397

398

399

400

401

402
Feng Z, and Levine AJ. 2010. The regulation of energy metabolism and the IGF-1/mTOR pathways by the p53 protein. Trends in Cell Biology 20:427-434. 10.1016/j.tcb.2010.03.004

Fields AP, and Justilien V. 2009. The guanine nucleotide exchange factor (GEF) Ect2 is an oncogene in human cancer. Advances in Enzyme Regulation 50:190-200. 10.1016/j.advenzreg.2009.10.010

Fuji T, Nomoto S, Koshikawa K, Yatabe Y, Teshigawara O, Mori T, Inoue S, Takeda S, and Nakao A. 2006. Overexpression of pituitary tumor transforming gene 1 in $\mathrm{HCC}$ is associated with angiogenesis and poor prognosis. Hepatology 43:1267-1275. 10.1002/hep.21181

Furukawa T, Kanai N, Shiwaku HO, Soga N, Uehara A, and Horii A. 2006. AURKA is one of the downstream targets of MAPK1/ERK2 in pancreatic cancer. Oncogene 25:4831-4839. 10.1038/sj.onc. 1209494

Goodarzi H, Liu X, Nguyen HCB, Zhang S, Fish L, and Tavazoie SF. 2015. Endogenous tRNA-Derived Fragments Suppress Breast Cancer Progression via YBX1 Displacement. Cell 161:790-802. 10.1016/j.cell.2015.02.053

Hua S, Ji Z, Quan Y, Zhan M, Wang H, Li W, Li Y, He X, and Lu L. 2020. Identification of hub genes in hepatocellular carcinoma using integrated bioinformatic analysis. Aging 12:5439-5468. 10.18632/aging. 102969

Hussain SP, Schwank J, Staib F, Wang XW, and Harris CC. 2007. TP53 mutations and hepatocellular carcinoma: insights into the etiology and pathogenesis of liver cancer. Oncogene 26:2166-2176. 10.1038/sj.onc. 1210279

Hutter C, and Zenklusen JC. 2018. The Cancer Genome Atlas: Creating Lasting Value beyond Its Data. Cell 173:283-285. 10.1016/j.cell.2018.03.042

Ichikawa M, Arai Y, Haruta M, Furukawa S, Ariga T, Kajii T, and Kaneko Y. 2013. Meiosis error and subsequent genetic and epigenetic alterations invoke the malignant transformation of germ cell tumor. Genes Chromosomes Cancer 52:274-286. 10.1002/gcc.22027

Isaacs RJ, Davies SL, Sandri MI, Redwood C, Wells NJ, and Hickson ID. 1998. Physiological regulation of eukaryotic topoisomerase II. Biochim Biophys Acta 1400:121-137. 10.1016/s01674781(98)00131-6

Jaenisch R, and Bird A. 2003. Epigenetic regulation of gene expression: how the genome integrates intrinsic and environmental signals. Nature Genetics 33 Suppl:245-254. 10.1038/ng1089

Jarrard DF, Kinoshita H, Shi Y, Sandefur C, Hoff D, Meisner LF, Chang C, Herman JG, Isaacs WB, and Nassif N. 1998. Methylation of the androgen receptor promoter CpG island is associated with loss of androgen receptor expression in prostate cancer cells. Cancer Research 58:5310-5314.

Jiang CH, Yuan X, Li JF, Xie YF, Zhang AZ, Wang XL, Yang L, Liu CX, Liang WH, Pang LJ, Zou H, Cui XB, Shen XH, Qi Y, Jiang JF, Gu WY, Li F, and Hu JM. 2020. Bioinformatics-based screening of key genes for transformation of liver cirrhosis to hepatocellular carcinoma. Journal of Translational Medicine 18:40. 10.1186/s12967-020-02229-8

Peer) reviewing PDF | (2020:06:49658:3:0:NEW 24 Nov 2020) 
403

404

405

406

407

408

409

410

411

412

413

414

415

416

417

418

419

420

421

422

423

424

425

426

427

428

429

430

431

432

433

434

435

436

437

438

439

Jiang P, Tang Y, He L, Tang H, Liang M, Mai C, Hu L, and Hong J. 2014. Aberrant expression of nuclear KPNA2 is correlated with early recurrence and poor prognosis in patients with small hepatocellular carcinoma after hepatectomy. Medical Oncology 31:131. 10.1007/s12032-0140131-4

Li C, Qin F, Hong H, Tang H, Jiang X, Yang S, Mei Z, and Zhou D. 2019. Identification of Flap endonuclease 1 as a potential core gene in hepatocellular carcinoma by integrated bioinformatics analysis. PeerJ 7:e7619. 10.7717/peerj.7619

Li J, Gao JZ, Du JL, Huang ZX, and Wei LX. 2014. Increased CDC20 expression is associated with development and progression of hepatocellular carcinoma. International Journal of Oncology 45:1547-1555. 10.3892/ijo.2014.2559

Li X, Xiong X, Zhang M, Wang K, Chen Y, Zhou J, Mao Y, Lv J, Yi D, Chen XW, Wang C, Qian SB, and Yi C. 2017. Base-Resolution Mapping Reveals Distinct m(1)A Methylome in Nuclear- and Mitochondrial-Encoded Transcripts. Molecular Cell 68:993-1005.e1009. 10.1016/j.molcel.2017.10.019

Liang HW, Yang X, Wen DY, Gao L, Zhang XY, Ye ZH, Luo J, Li ZY, He Y, Pang YY, and Chen G. 2018. Utility of miR133a3p as a diagnostic indicator for hepatocellular carcinoma: An investigation combined with GEO, TCGA, metaanalysis and bioinformatics. Mol Med Rep 17:1469-1484. 10.3892/mmr.2017.8040

Liu M, Zhang Y, Liao Y, Chen Y, Pan Y, Tian H, Zhan Y, and Liu D. 2015. Evaluation of the Antitumor Efficacy of RNAi-Mediated Inhibition of CDC20 and Heparanase in an Orthotopic Liver Tumor Model. Cancer Biother Radiopharm 30:233-239. 10.1089/cbr.2014.1799

Liu Z, Li J, Chen J, Shan Q, Dai H, Xie H, Zhou L, Xu X, and Zheng S. 2018. MCM family in HCC: MCM6 indicates adverse tumor features and poor outcomes and promotes $\mathrm{S} / \mathrm{G} 2$ cell cycle progression. BMC Cancer 18:200. 10.1186/s12885-018-4056-8

Madine MA, Khoo CY, Mills AD, and Laskey RA. 1995. MCM3 complex required for cell cycle regulation of DNA replication in vertebrate cells. Nature 375:421-424. 10.1038/375421a0

Musahl C, Holthoff HP, Lesch R, and Knippers R. 1998. Stability of the Replicative Mcm3 Protein in Proliferating and Differentiating Human Cells. Experimental Cell Research 241:0-264. 10.1006/excr.1998.4041

Newman MEJ, and Girvan M. 2004. Finding and Evaluating Community Structure in Networks. Physical Review E Statistical Nonlinear \& Soft Matter Physics 69:026113. 10.1103/PhysRevE.69.026113

Ni FB, Lin Z, Fan XH, Shi KQ, Ao JY, Wang XD, and Chen RC. 2020. A novel genomicclinicopathologic nomogram to improve prognosis prediction of hepatocellular carcinoma. Clinica Chimica Acta 504:88-97. 10.1016/j.cca.2020.02.001

Nientiedt M, Deng M, Schmidt D, Perner S, Müller SC, and Ellinger Jr. 2016. Identification of aberrant tRNA-halves expression patterns in clear cell renal cell carcinoma. Scientific Reports 6:37158. $10.1038 /$ srep37158

Peer) reviewing PDF | (2020:06:49658:3:0:NEW 24 Nov 2020) 
440

441

442

443

444

445

446

447

448

449

450

451

452

453

454

455

456

457

458

459

460

461

462

463

464

465

466

467

468

469

470

471

472

473

474

475

Ohtani-Fujita N, Fujita T, Aoike A, Osifchin NE, Robbins PD, and Sakai T. 1993. CpG methylation inactivates the promoter activity of the human retinoblastoma tumor-suppressor gene. Oncogene 8:1063-1067.

Panvichian R, Tantiwetrueangdet A, Angkathunyakul N, and Leelaudomlipi S. 2015. TOP2A amplification and overexpression in hepatocellular carcinoma tissues. Biomed Res Int 2015:381602. 10.1155/2015/381602

Phillippa, Taberlay, Joanna, Achinger-Kawecka, Aaron, Lun, and Fabian. 2016. Three-dimensional disorganization of the cancer genome occurs coincident with long-range genetic and epigenetic alterations. Genome Research. 10.1101/gr.201517.115

Raghavan UN, Albert R, and Kumara S. 2007. Near linear time algorithm to detect community structures in large-scale networks. Physical Review E Statistical Nonlinear \& Soft Matter Physics 76:036106. 10.1103/PhysRevE.76.036106

Shannon P, Markiel A, Ozier O, Baliga NS, Wang JT, Ramage D, Amin N, Schwikowski B, and Ideker T. 2003. Cytoscape: a software environment for integrated models of biomolecular interaction networks. Genome Research 13:2498-2504. 10.1101/gr.1239303

Shen-Jie JI, and Li G. 2018. The diagnostic value of joint detection of serum AFP,CA125 and TK1 in patients with primary hepatic carcinoma. Journal of Tropical Medicine.

Song H, Ding N, Li S, Liao J, Xie A, Yu Y, Zhang C, and Ni C. 2020. Identification of Hub Genes Associated With Hepatocellular Carcinoma Using Robust Rank Aggregation Combined With Weighted Gene Co-expression Network Analysis. Front Genet 11:895. 10.3389/fgene. 2020.00895

Szklarczyk D, Franceschini A, Kuhn M, Simonovic M, Roth A, Minguez P, Doerks T, Stark M, Muller J, Bork P, Jensen LJ, and von Mering C. 2011. The STRING database in 2011: functional interaction networks of proteins, globally integrated and scored. Nucleic Acids Research 39:D561-568. 10.1093/nar/gkq973

Tatsumoto, and T. 1999. Human ECT2 Is an Exchange Factor for Rho GTPases, Phosphorylated in G2/M Phases, and Involved in Cytokinesis. Journal of Cell Biology 147:921-928. 10.1083/jcb.147.5.921

Vogelstein B, Lane D, and Levine AJ. 2000. Surfing the p53 network. Nature 408:307-310. $10.1038 / 35042675$

Wang Y, Huang Q, Deng T, Li BH, and Ren XQ. 2019. Clinical Significance of TRMT6 in Hepatocellular Carcinoma: A Bioinformatics-Based Study. Medical Science Monitor 25:38943901. 10.12659/msm.913556

Wilkinson KA, and Henley JM. 2010. Mechanisms, regulation and consequences of protein SUMOylation. Biochemical Journal 428:133-145. 10.1042/BJ20100158

Wold S, Esbensen K, and Geladi P. 1987. Principal component analysis. Chemometrics and Intelligent Laboratory Systems 2:37-52.

Peer) reviewing PDF | (2020:06:49658:3:0:NEW 24 Nov 2020) 
476

477

478

479

480

481

482

483

484

485

486

487

488

489

490

491

492

493

494

495

496

497

498

499

500

501

502

503

504

505

506

507

508

509
Wong N, Yeo W, Wong WL, Wong NL, Chan KY, Mo FK, Koh J, Chan SL, Chan AT, Lai PB, Ching AK, Tong JH, Ng HK, Johnson PJ, and To KF. 2009. TOP2A overexpression in hepatocellular carcinoma correlates with early age onset, shorter patients survival and chemoresistance. International Journal of Cancer 124:644-652. 10.1002/ijc.23968

Xinggang G, Zhiheng W, Jianing Z, Qingguo X, Guojun H, Yuan Y, Chuanpeng D, Gang L, Chenhua L, and Lei L. 2019. Upregulated KPNA2 promotes hepatocellular carcinoma progression and indicates prognostic significance across human cancer types. Acta Biochimica et Biophysica Sinica:3. 10.1093/abbs/gmz003

Yan H, Merchant AM, and Tye BK. 1993. Cell cycle-regulated nuclear localization of MCM2 and MCM3, which are required for the initiation of DNA synthesis at chromosomal replication origins in yeast. Genes Dev 7:2149-2160. 10.1101/gad.7.11.2149

Yang WX, Pan YY, and You CG. 2019. CDK1, CCNB1, CDC20, BUB1, MAD2L1, MCM3, BUB1B, MCM2, and RFC4 May Be Potential Therapeutic Targets for Hepatocellular Carcinoma Using Integrated Bioinformatic Analysis. Biomed Res Int 2019:1245072. 10.1155/2019/1245072

Yue C, Liang C, Li P, Yan L, Zhang D, Xu Y, Wei Z, and Wu J. 2019. DUXAP8 a Pan-Cancer Prognostic Marker Involved in the Molecular Regulatory Mechanism in Hepatocellular Carcinoma: A Comprehensive Study Based on Data Mining, Bioinformatics, and in vitro Validation. OncoTargets and Therapy 12:11637-11650. 10.2147/ott.S231750

Zhang Q, Sun S, Zhu C, Zheng Y, Cai Q, Liang X, Xie H, and Zhou J. 2019. Prediction and analysis of weighted genes in hepatocellular carcinoma using bioinformatics analysis. Mol Med Rep 19:2479-2488. 10.3892/mmr.2019.9929

Zhang SY, Lin BD, and Li BR. 2015. Evaluation of the diagnostic value of alpha-1-fucosidase, alphafetoprotein and thymidine kinase 1 with ROC and logistic regression for hepatocellular carcinoma. FEBS Open Bio 5:240-244. 10.1016/j.fob.2015.03.010

Zhao Y, Wang Y, Zhu F, Zhang J, Ma X, and Zhang D. 2020. Gene expression profiling revealed MCM3 to be a better marker than Ki67 in prognosis of invasive ductal breast carcinoma patients. Clinical and Experimental Medicine 20:249-259. 10.1007/s10238-019-00604-4

Zhong J, Agha G, and Baccarelli AA. 2016. The Role of DNA Methylation in Cardiovascular Risk and Disease: Methodological Aspects, Study Design, and Data Analysis for Epidemiological Studies. Circulation Research 118:119-131. 10.1161/circresaha.115.305206

Zhuang L, Yang Z, and Meng Z. 2018. Upregulation of BUB1B, CCNB1, CDC7, CDC20, and MCM3 in Tumor Tissues Predicted Worse Overall Survival and Disease-Free Survival in Hepatocellular Carcinoma Patients. Biomed Res Int 2018:7897346. 10.1155/2018/7897346

Peer) reviewing PDF | (2020:06:49658:3:0:NEW 24 Nov 2020) 
Figure 1

Flow-chart of data analysis in this paper.

Downloading data from TCGA and preprocessing data

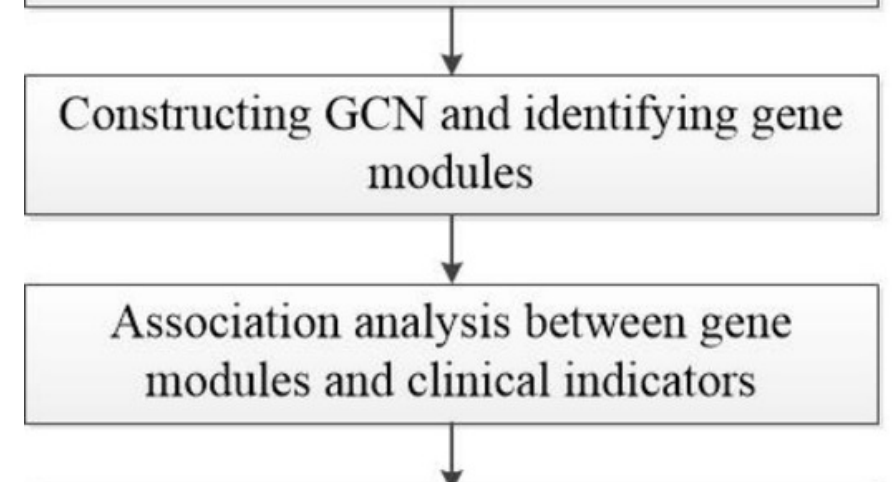

Identifying key gene module and verify the importance of them

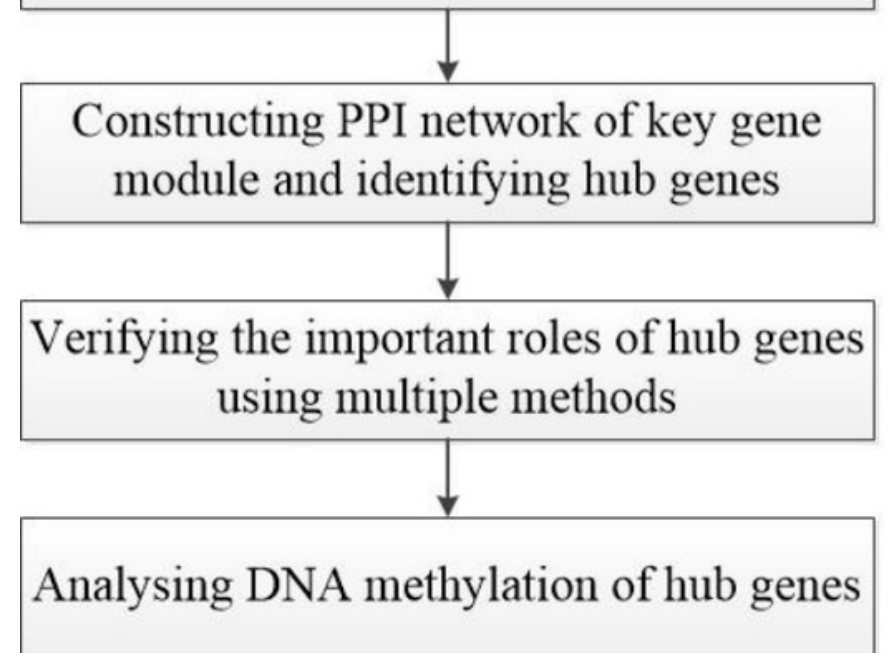

$\mathrm{GO} /$ Reactome enrichment analysis of key gene module 
Figure 2

Identification results of DEGs.

(A) $\mathrm{X}$-axis represents log2 fold-changes and $\mathrm{Y}$-axis represents negative logarithm to the base 10 of the $P$-values. Black vertical and horizontal dashed lines reflect filtering criteria $(\log 2$ (Fold change) $= \pm 1$ and $P$-value $=0.05)$. (B) Pink and green bars are number of significantly up-regulated $(n=4,130)$ and down-regulated genes $(n=471)$ in HCC compared with its normal tissues.
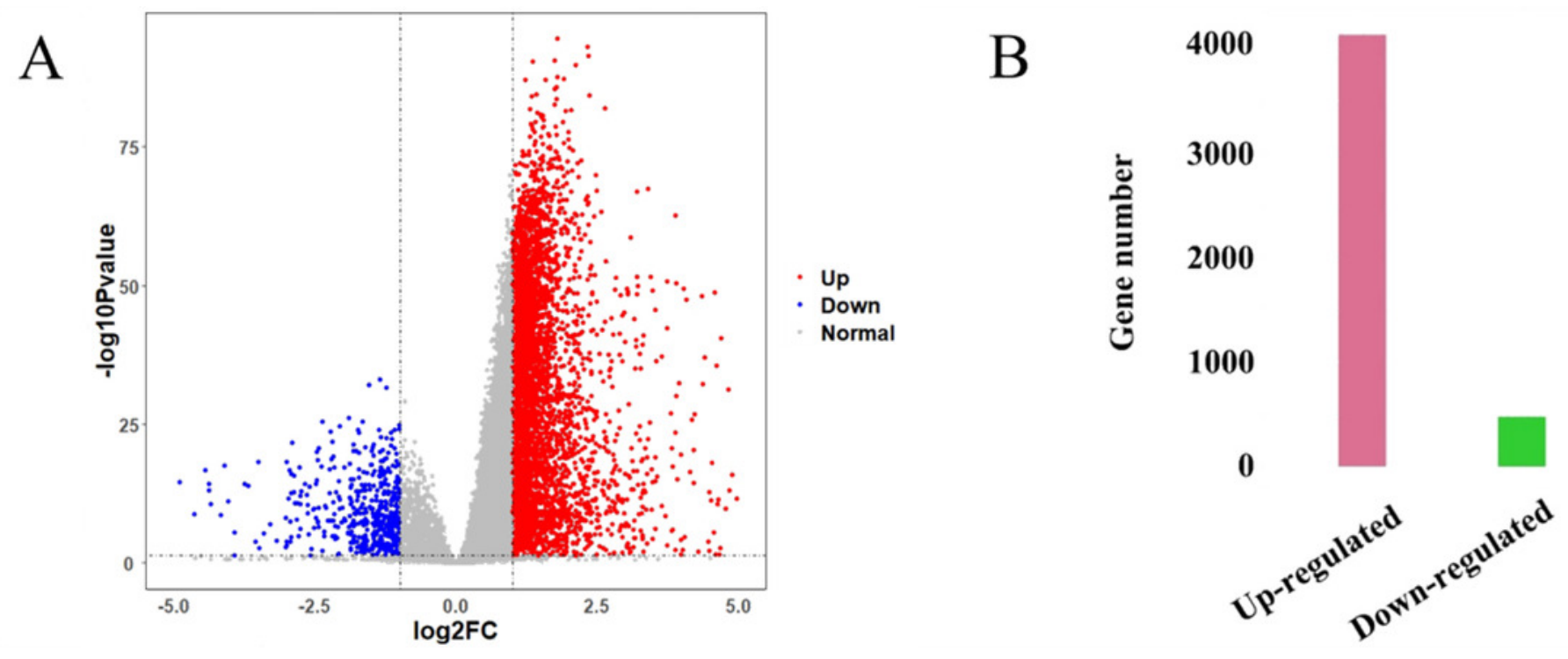
Figure 3

The GCN was constructed by Pearson correlation analysis.

The total number of gene in figure is 2859 , and the number of gene in GCN (the largest network) is 2583 .

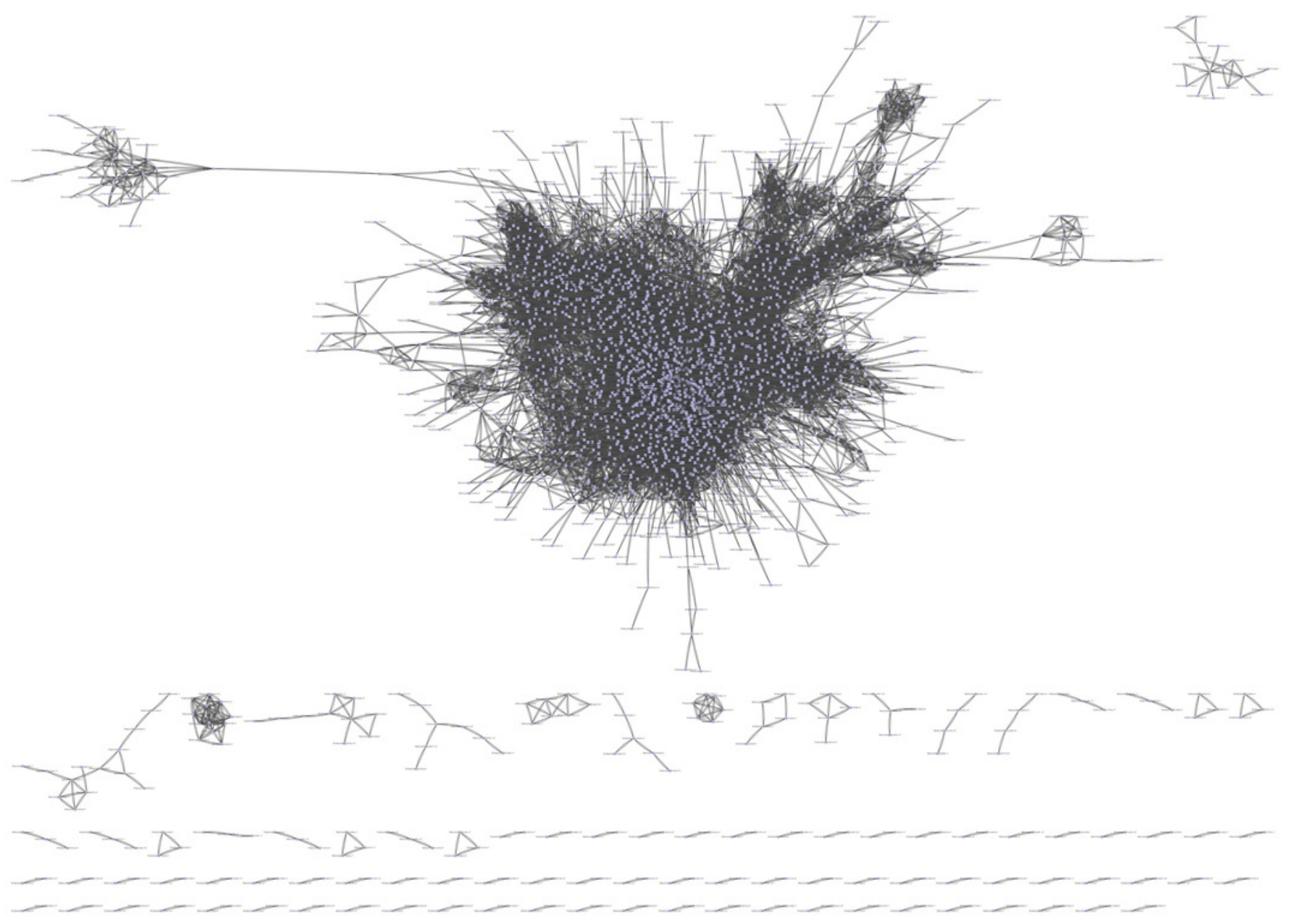


Figure 4

Module identification result obtained by the multilevel algorithm.

The multilevel algorithm divided GCN into 13 gene modules, the little modules with less than 50 genes were removed, and the labeled modules were 9 gene modules for further analysis.

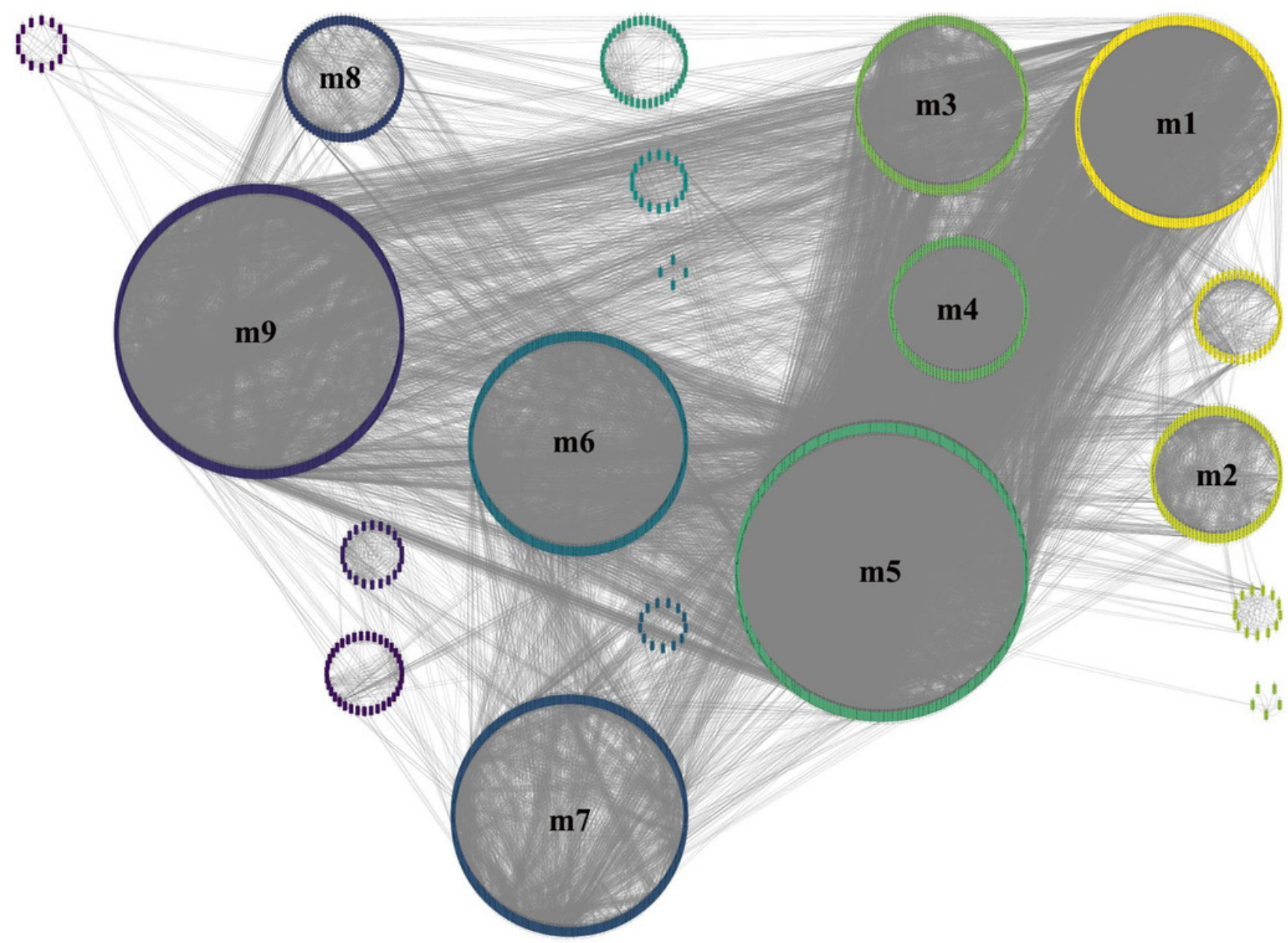




\section{Figure 5}

The heat map of the correlation between gene modules and clinical indicators.

The row corresponds to module, and the column corresponds to clinical indicator. The $\mathrm{ml}$ is key gene module in HCC.

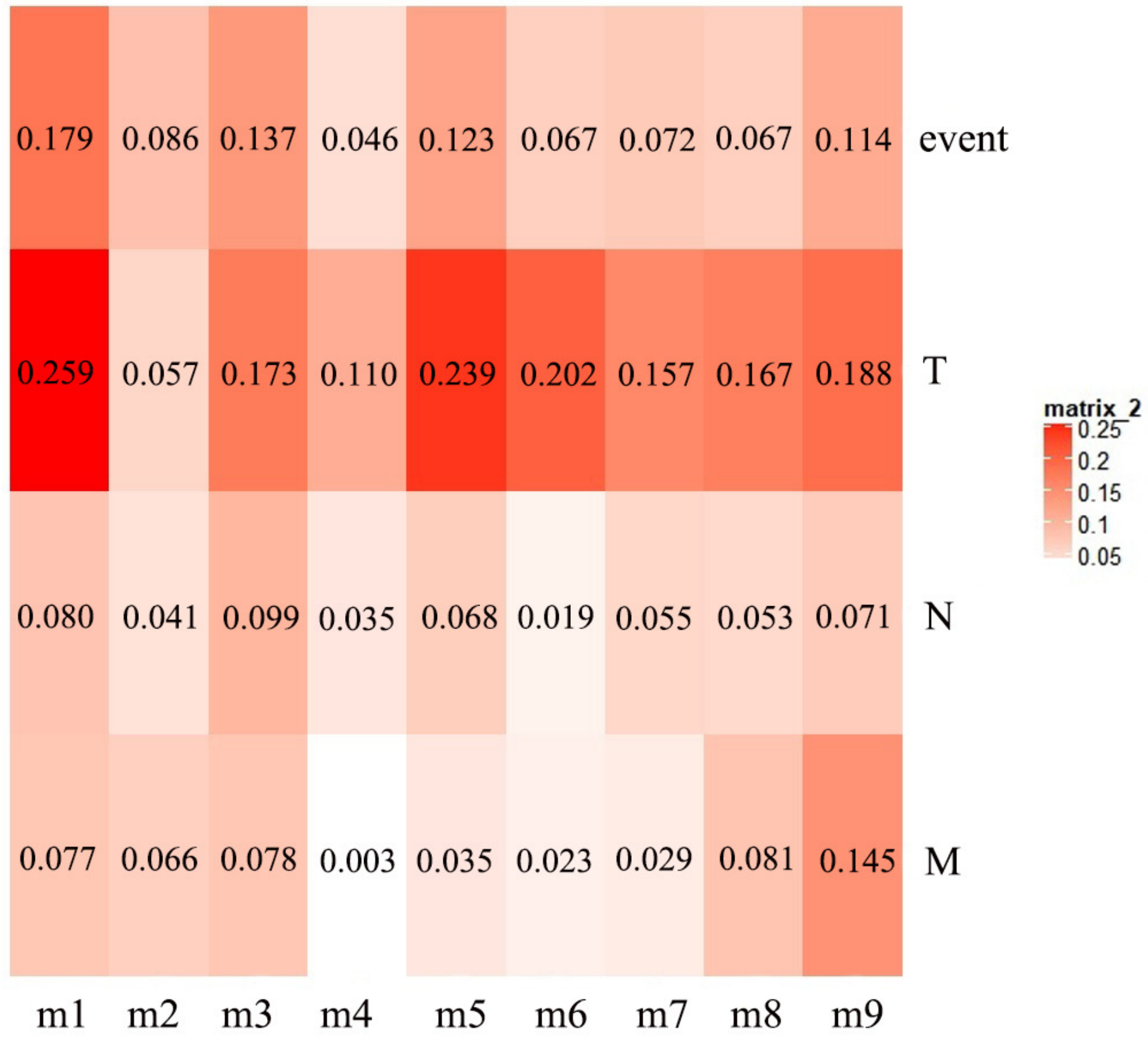


Figure 6

The PS-values of all modules.

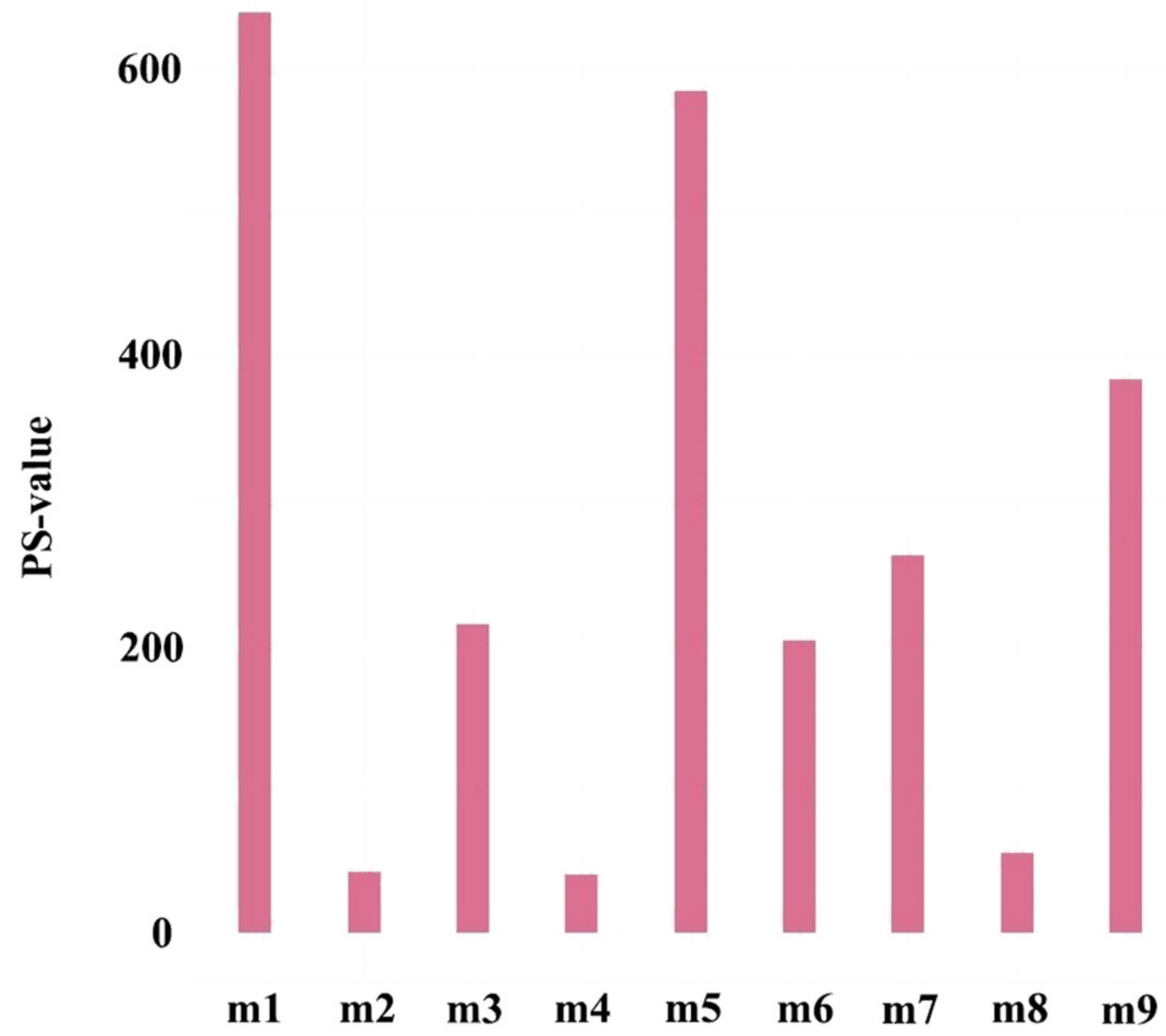




\section{Figure 7}

The $20 \mathrm{GO}$ Terms (biological processes) with the smallest $P$-value of genes in the key gene module (m1).

The size of bubbles represents the numbers of genes, the color of bubbles corresponds to $P$ value, and the GeneRatio represents the ratio of the number of genes enriched to the total number of genes in key gene module (m1).

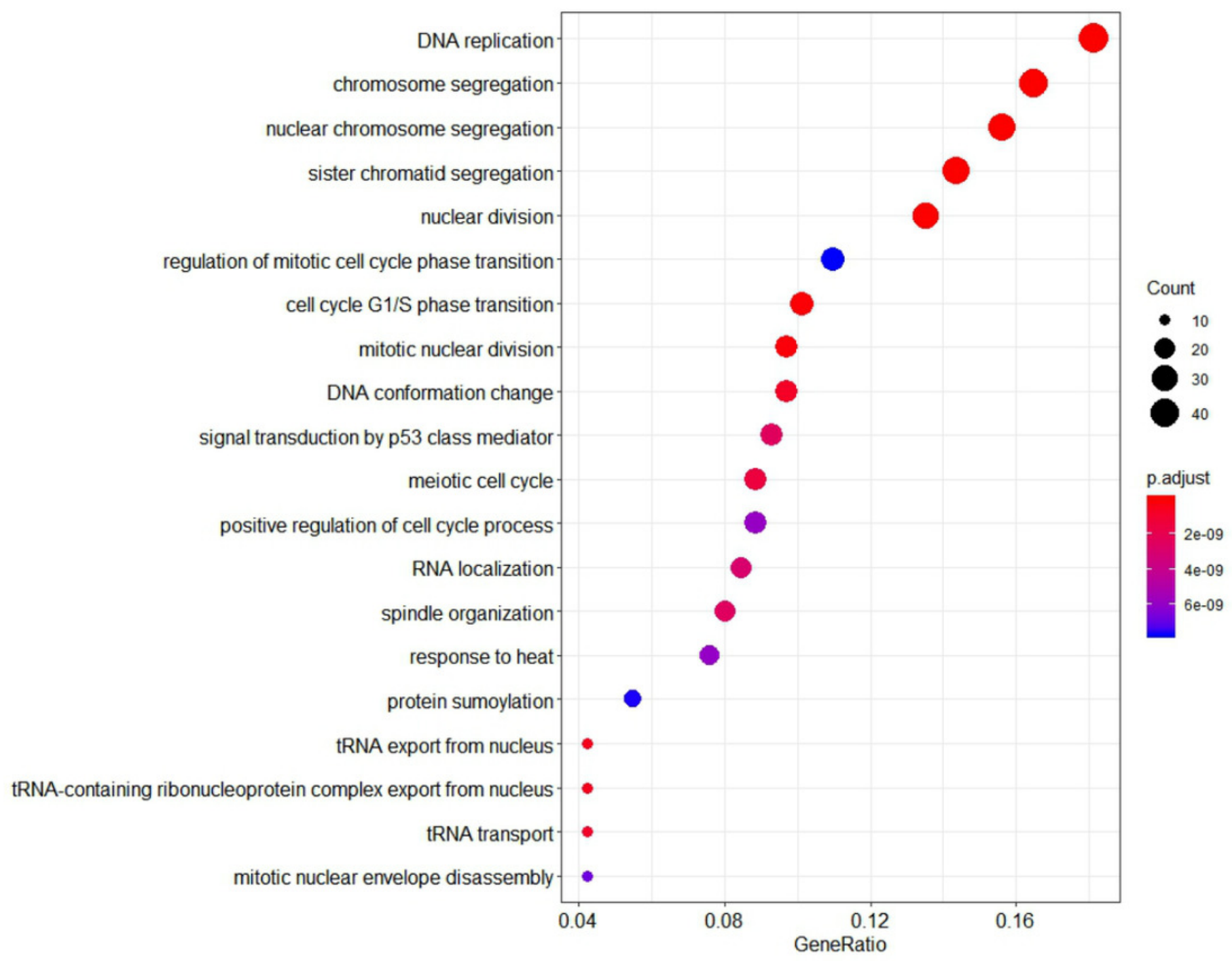




\section{Figure 8}

The PPI network of key gene module (m1).

Each node corresponds to a gene, where a red node corresponds to a hub gene.

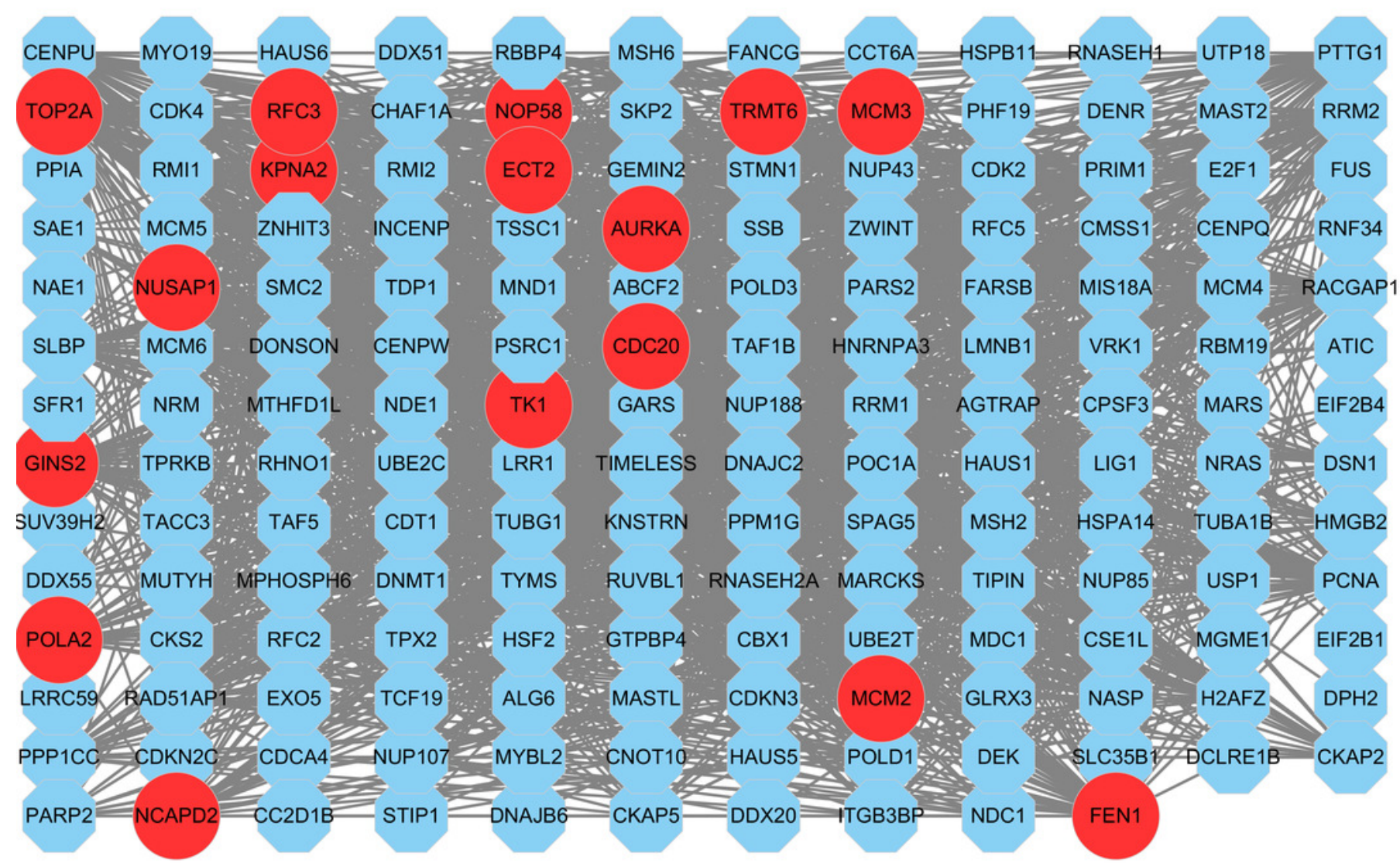


Figure 9

The results returned from Oncomine database.

The row corresponds to cancer, and the column corresponds to gene. The red square represents that the gene was up-regulated in cancer, the blue square represents that the gene was down-regulated in cancer, and the value in the square represents the number of related references.

\begin{tabular}{|c|c|c|c|c|c|c|c|c|c|c|c|c|c|c|c|c|c|c|c|c|c|c|c|c|c|c|c|c|c|c|c|c|}
\hline \multirow{2}{*}{$\begin{array}{l}\text { Analysis Type by Cancer } \\
\text { Bladder Cancer }\end{array}$} & \multicolumn{2}{|c|}{\begin{tabular}{|c}
$\begin{array}{c}\text { Cancer } \\
\text { vs. } \\
\text { Normal } \\
\text { AURKA }\end{array}$ \\
\end{tabular}} & \multicolumn{2}{|c|}{$\begin{array}{c}\text { Cancer } \\
\text { vs. } \\
\text { Normal } \\
\text { CDC20 }\end{array}$} & \multicolumn{2}{|c|}{ 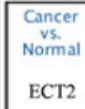 } & \multicolumn{2}{|c|}{$\begin{array}{c}\begin{array}{c}\text { Cancer } \\
\text { vs. } \\
\text { Normal } \\
\text { FEN1 }\end{array} \\
\end{array}$} & \multicolumn{2}{|c|}{ 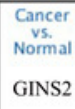 } & \multicolumn{2}{|c|}{$\begin{array}{c}\begin{array}{c}\text { Cancer } \\
\text { vs. } \\
\text { Normal }\end{array} \\
\text { KPNA2 }\end{array}$} & \multicolumn{2}{|c|}{$\begin{array}{c}\text { Cancer } \\
\text { vs. } \\
\text { Normal } \\
\text { MCM2 }\end{array}$} & \multicolumn{2}{|c|}{$\begin{array}{c}\begin{array}{c}\text { Cancer } \\
\text { vs. } \\
\text { Normal }\end{array} \\
\text { MCM3 }\end{array}$} & \multicolumn{2}{|c|}{\begin{tabular}{|c}
$\begin{array}{c}\text { Cancer } \\
\text { vs. } \\
\text { Normal } \\
\text { NCAPD2 }\end{array}$ \\
NCAP
\end{tabular}} & \multicolumn{2}{|c|}{$\begin{array}{c}\text { Cancer } \\
\text { vs. } \\
\text { Normal } \\
\text { NOP58 }\end{array}$} & \multicolumn{2}{|c|}{$\begin{array}{l}\text { Cancer } \\
\text { vs. } \\
\text { Normal } \\
\text { NUSAP1 }\end{array}$} & \multicolumn{2}{|c|}{$\begin{array}{c}\begin{array}{c}\text { Cancer } \\
\text { vs. } \\
\text { Normal } \\
\text { POLA2 }\end{array} \\
\end{array}$} & \multicolumn{2}{|c|}{$\begin{array}{c}\text { Cancer } \\
\text { vs. } \\
\text { Normal } \\
\text { RFC3 }\end{array}$} & \multicolumn{2}{|c|}{$\begin{array}{c}\text { Cancer } \\
\text { vs. } \\
\text { Normal } \\
\text { TK1 }\end{array}$} & \multicolumn{2}{|c|}{\begin{tabular}{|c}
$\begin{array}{c}\text { Cancer } \\
\text { vs. } \\
\text { Normal } \\
\text { TOP2A }\end{array}$ \\
TOP
\end{tabular}} & \multicolumn{2}{|c|}{$\begin{array}{l}\text { Cancer } \\
\text { vs. } \\
\text { Normal } \\
\text { TRMT6 }\end{array}$} \\
\hline & 5 & & 6 & & 2 & & 6 & & 3 & & 5 & & 3 & & 3 & & 1 & & & & 5 & & 2 & & & & 3 & & 8 & & & \\
\hline Brain and CNS Cancer & 3 & 1 & 5 & & 6 & & & 1 & 4 & 1 & 3 & 1 & 6 & 1 & 6 & 1 & 3 & & & & 10 & & & 1 & 3 & 1 & 1 & 1 & 12 & & & \\
\hline Breast Cancer & 21 & 1 & 19 & & 19 & 1 & 11 & 1 & 20 & 1 & 18 & 1 & 8 & & 4 & & 3 & 1 & & 1 & 23 & & 1 & & & & 21 & 1 & 28 & 1 & 2 & \\
\hline Cervical Cancer & 3 & & 3 & & 4 & & 4 & & 4 & & 2 & & 4 & & 3 & & 1 & & & & 4 & & 2 & & 3 & & 3 & & 4 & & 1 & \\
\hline Colorectal Cancer & 16 & & 6 & & 22 & & 7 & & 7 & & 12 & & 10 & & 6 & & 5 & & 6 & & 11 & & 3 & & 19 & & 3 & & 19 & & 11 & \\
\hline Esophageal Cancer & 3 & & 2 & & 3 & & 2 & & 1 & & 2 & & 2 & & 2 & & 1 & & & & 2 & & 2 & & 1 & & 1 & & 2 & & 1 & \\
\hline Gastric Cancer & 4 & & 2 & & 8 & & 2 & & 4 & & 6 & & 2 & & 2 & & 1 & & 1 & & 5 & & 1 & & 4 & & & & 10 & & 2 & \\
\hline Head and Neck Cancer & 10 & & 8 & & 10 & & 3 & & 3 & & 9 & & 9 & & 2 & & 3 & & & & 3 & & & & 3 & & 7 & & 10 & & 2 & \\
\hline Kidney Cancer & & 2 & 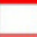 & & 2 & 1 & 2 & & 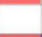 & & 1 & & 4 & & & 1 & & & & & 3 & & & 2 & 1 & & & 2 & 3 & & & \\
\hline Leukemia & 1 & 7 & 1 & 5 & 2 & 1 & & 2 & 1 & 3 & 1 & 1 & 1 & 1 & 1 & 1 & & 2 & & & 2 & 3 & & & 3 & 5 & 1 & 2 & 1 & 5 & & \\
\hline Liver Cancer & 4 & & 3 & & 3 & & 3 & & & & 4 & & 2 & & 3 & & 1 & & & & 5 & & & & 1 & & 1 & & 4 & & 1 & \\
\hline Lung Cancer & 14 & & 12 & & 7 & & 12 & & 7 & & 14 & & 9 & & 6 & & 4 & & & & 11 & & 1 & & 4 & & 15 & & 21 & & & \\
\hline Lymphoma & 5 & 1 & 8 & 1 & 4 & & 8 & & 4 & & 1 & & & & 4 & & 3 & & & & 5 & & 1 & & 5 & & 8 & & 9 & & & \\
\hline Melanoma & & & 1 & & & & 1 & & & & 2 & & & & 2 & & 1 & & & & 3 & & & & 1 & & & & 3 & 1 & & \\
\hline Myeloma & & & & 2 & 1 & & 1 & & & 1 & 1 & & 1 & & 1 & & & & & & & & & & & & & & & & & \\
\hline Other Cancer & 3 & 1 & 5 & 2 & 4 & 1 & 5 & & 3 & & 5 & 5 & 8 & & 5 & & 4 & & 3 & & 8 & & 2 & 1 & 4 & & 5 & & 11 & 1 & 1 & 1 \\
\hline Ovarian Cancer & 3 & & 4 & & 5 & & 1 & & 2 & & 7 & & 3 & & 1 & & 3 & & 2 & & 3 & & 1 & & 1 & & 3 & & 6 & & & \\
\hline Pancreatic Cancer & 1 & 1 & 2 & & 4 & 1 & 1 & & 1 & & 3 & & 1 & & & & & & & & 3 & & & & & & 1 & 1 & 5 & & 1 & \\
\hline Prostate Cancer & 1 & & & & 1 & & & & & & 1 & & & & & 1 & & & & & 3 & & & & & & 1 & & 3 & & & \\
\hline Sarcoma & 9 & 1 & 12 & 1 & 9 & & 9 & & 11 & & 9 & & 13 & & 10 & 1 & 6 & & & & 11 & & 2 & & 10 & & 6 & & 12 & & & \\
\hline Significant Unique Analyses & 105 & 15 & 97 & 11 & 114 & 5 & 77 & 4 & 74 & 6 & 104 & 8 & 85 & 2 & 60 & 5 & 39 & 3 & 12 & 1 & 118 & 3 & 18 & 4 & 61 & 6 & 79 & 7 & 169 & 8 & 22 & 1 \\
\hline Total Unique Analyses & 4 & & 4 & & 34 & & 3 & & 37 & & 44 & 45 & 4 & & 4 & & 39 & & 2 & & 38 & & 44 & & 46 & & 4 & & 46 & & 29 & \\
\hline
\end{tabular}


Figure 10

Significant correlation between hub genes expression and survival.

Survival curves of genes AURKA, CDC20,ECT2, FEN1, etc. X-axis represents survival time and $\mathrm{Y}$-axis represents survival rate.

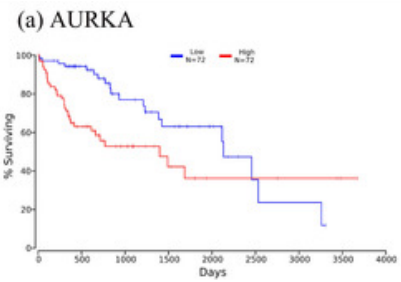

(e) GINS2
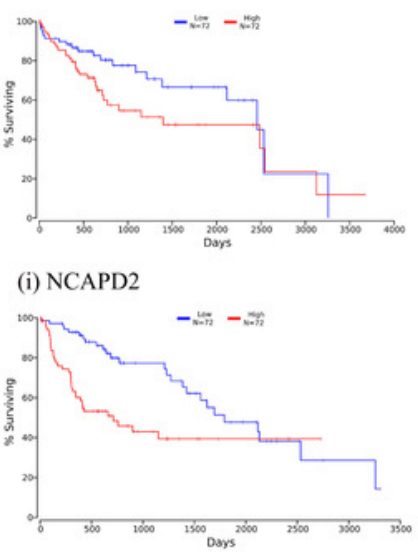

(m) RFC3

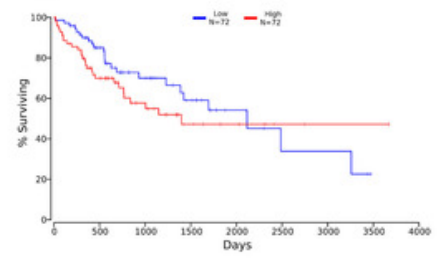

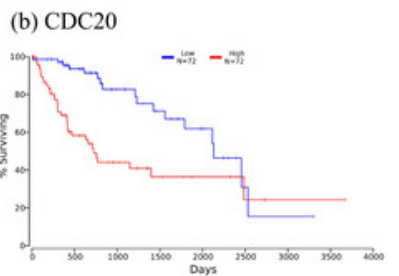

(f) KPNA2

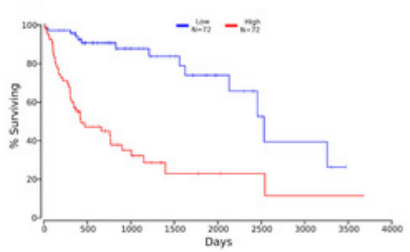

(j) NOP58

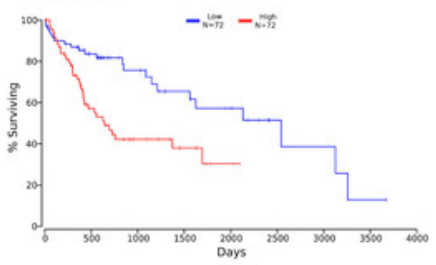

(n) TK1

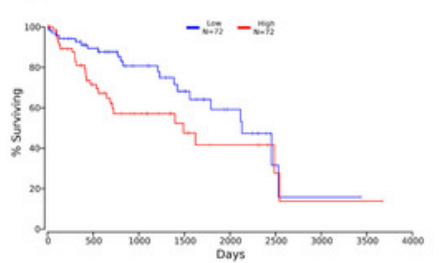

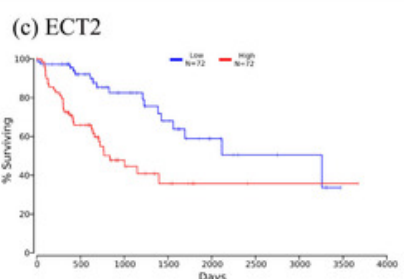
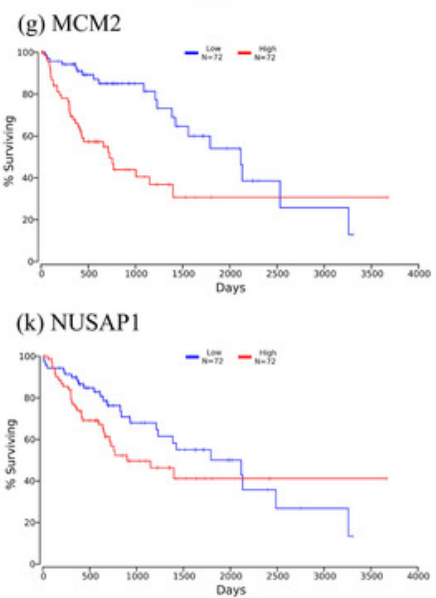

(o) TOP2A

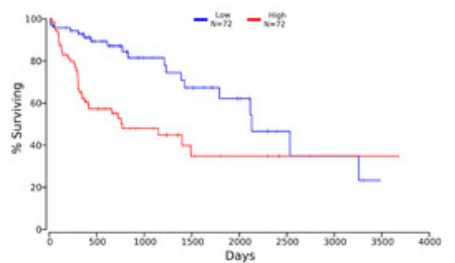

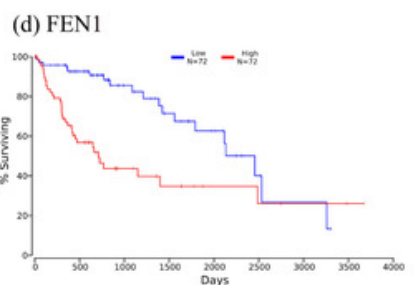

(h) $\mathrm{MCM} 3$
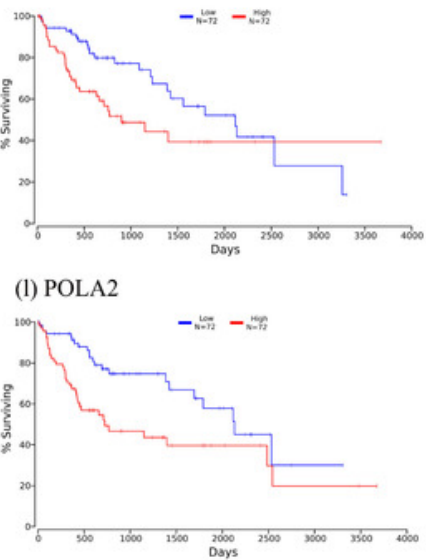

(p) TRMT6

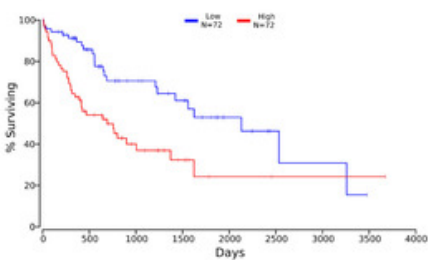




\section{Figure 11}

Te heat map of RPKM of KPNA2, MCM3, FEN1, TRMT6, ect. in normal and HCC samples.

TA-Tf represents HCC samples, NTA-NTf represents normal samples.

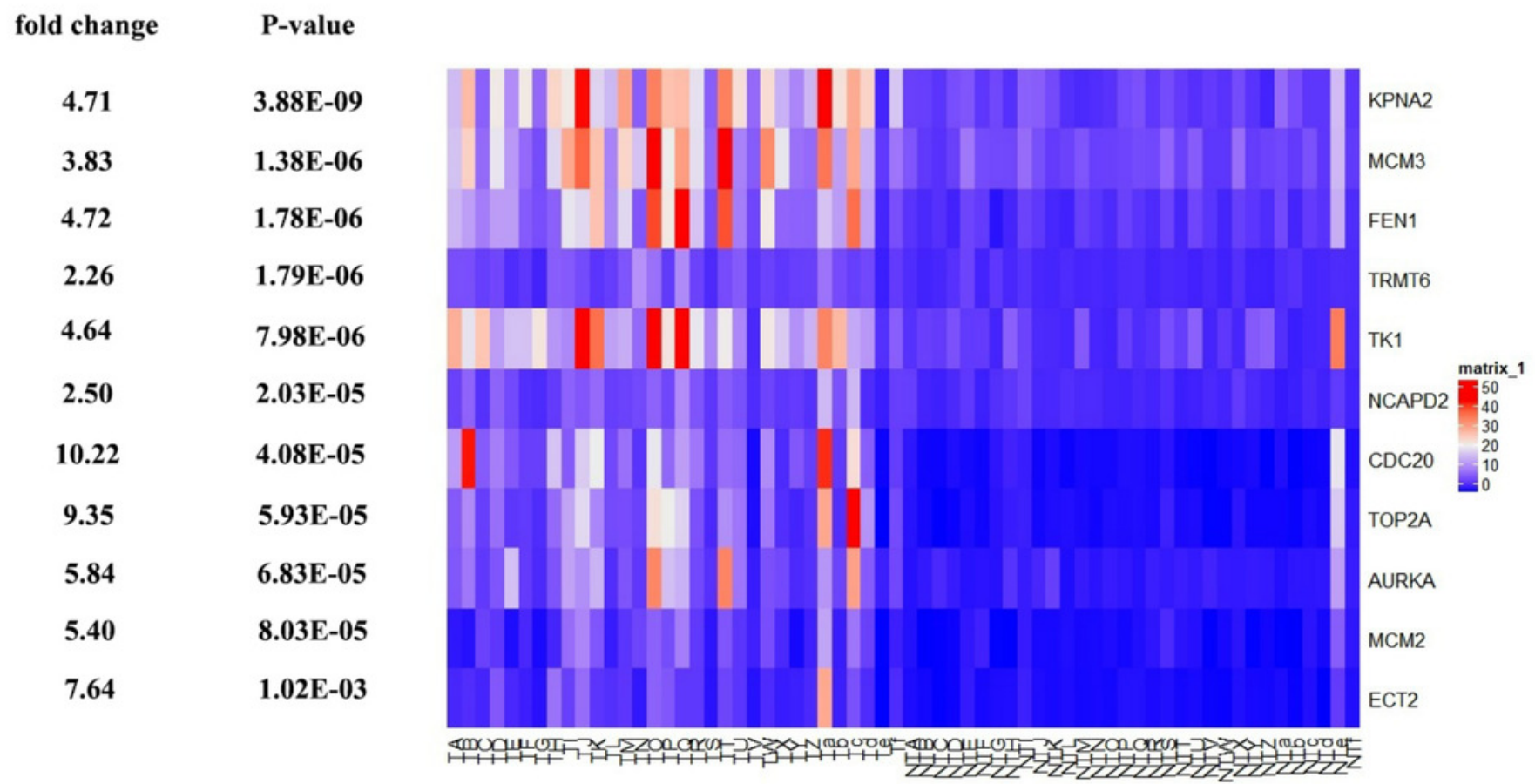


Figure 12

The ROC curves of 11 hub genes.

These ROC curves described the diagnostic efficiency of the mRNA levels of 11 hub genes for HCC and normal tissues.

(a) ARUKA

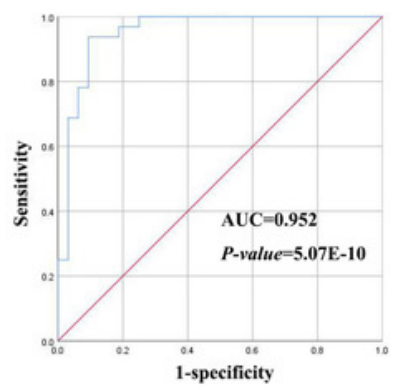

(e) KPNA2

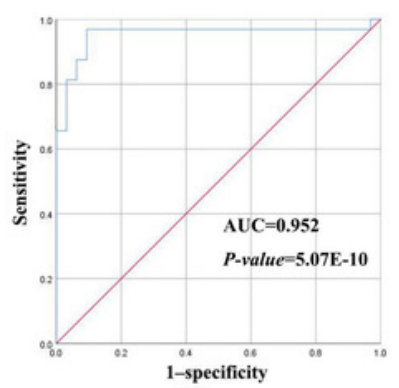

ตтх1

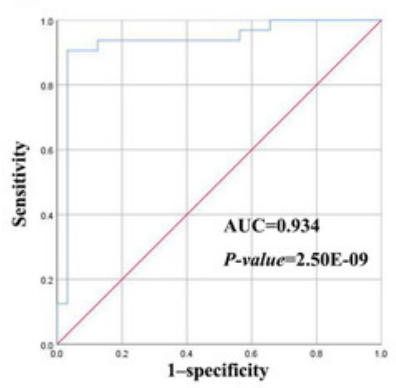

(b) CDC20

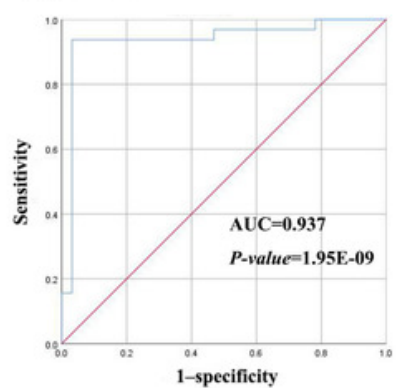

(f) MCM2

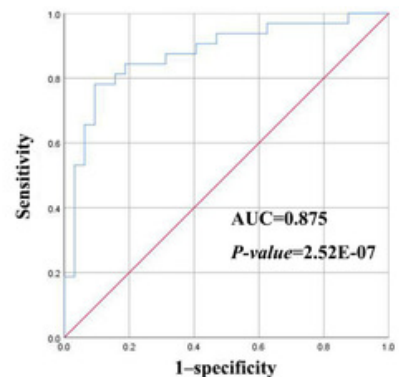

(j) TOP2A

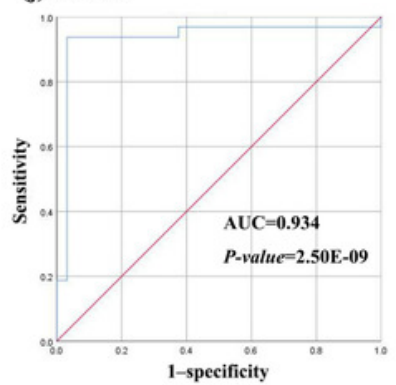

(c) ECT2

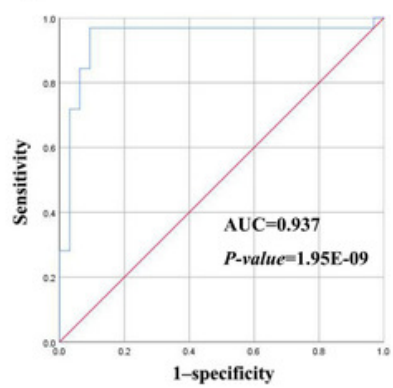

(g) $\mathrm{MCM}$

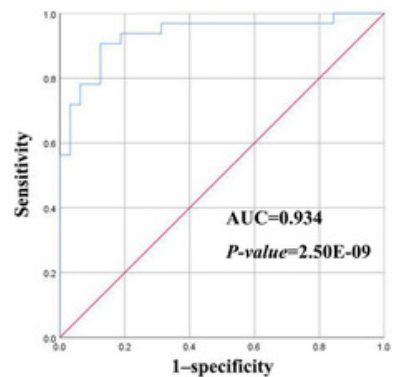

(k) TRMT6

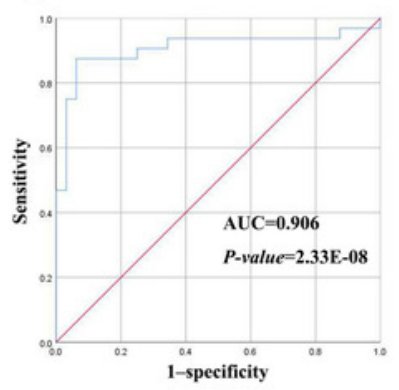

(d) FEN1

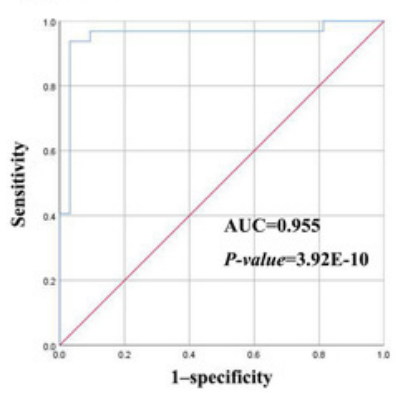

(h) NCAPD2

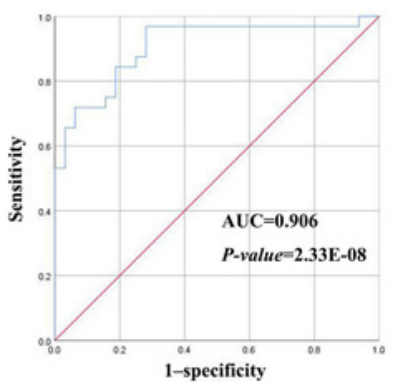




\section{Table 1 (on next page)}

The modularities of 3 algorithms. 


\begin{tabular}{cc}
\hline Algorithm & Modularity \\
\hline multilevel & 0.6009015 \\
label_propagation & 0.3748268 \\
edge-betweenness & 0.4815381 \\
\hline
\end{tabular}

1 
Table 2 (on next page)

The network densities of 9 gene modules containing more than 50 genes. 


\begin{tabular}{cc}
\hline Module & Densities \\
\hline $\mathrm{m} 1$ & 0.197628458 \\
$\mathrm{~m} 2$ & 0.29029703 \\
$\mathrm{~m} 3$ & 0.177997842 \\
$\mathrm{~m} 4$ & 0.215233881 \\
$\mathrm{~m} 5$ & 0.106922766 \\
$\mathrm{~m} 6$ & 0.090001227 \\
$\mathrm{~m} 7$ & 0.080307853 \\
$\mathrm{~m} 8$ & 0.216806723 \\
$\mathrm{~m} 9$ & 0.065630124 \\
\hline
\end{tabular}

1 


\section{Table 3 (on next page)}

The 20 signaling pathways with the smallest $P$-value of genes in the key gene module (m1). 


\begin{tabular}{|c|c|c|c|}
\hline ID & Description & Count & $P$-value \\
\hline R-HSA-69190 & DNA strand elongation & 18 & $1.11 \mathrm{E}-16$ \\
\hline R-HSA-453279 & Mitotic G1 phase and G1/S transition & 31 & $1.11 \mathrm{E}-16$ \\
\hline R-HSA-69278 & Cell Cycle, Mitotic & 78 & $1.11 \mathrm{E}-16$ \\
\hline $\begin{array}{l}\text { R-HSA- } \\
1640170\end{array}$ & Cell Cycle & 90 & $1.11 \mathrm{E}-16$ \\
\hline R-HSA-73894 & DNA Repair & 38 & $1.11 \mathrm{E}-16$ \\
\hline R-HSA-69620 & Cell Cycle Checkpoints & 35 & $1.11 \mathrm{E}-16$ \\
\hline R-HSA-69242 & S Phase & 27 & $2.22 \mathrm{E}-16$ \\
\hline R-HSA-69206 & G1/S Transition & 25 & $3.33 \mathrm{E}-16$ \\
\hline R-HSA-69306 & DNA Replication & 24 & $8.88 \mathrm{E}-16$ \\
\hline R-HSA-69239 & Synthesis of DNA & 23 & $2.33 \mathrm{E}-15$ \\
\hline R-HSA-68886 & M Phase & 36 & $8.33 \mathrm{E}-15$ \\
\hline R-HSA-73886 & Chromosome Maintenance & 20 & $3.18 \mathrm{E}-14$ \\
\hline $\begin{array}{l}\text { R-HSA- } \\
5693532\end{array}$ & DNA Double-Strand Break Repair & 24 & $3.45 \mathrm{E}-14$ \\
\hline $\begin{array}{l}\text { R-HSA- } \\
5693538\end{array}$ & Homology Directed Repair & 21 & $1.63 \mathrm{E}-13$ \\
\hline R-HSA-68877 & Mitotic Prometaphase & 24 & 4.54E-12 \\
\hline R-HSA-69186 & Lagging Strand Synthesis & 11 & $5.40 \mathrm{E}-12$ \\
\hline $\begin{array}{l}\text { R-HSA- } \\
5693567\end{array}$ & $\begin{array}{l}\text { HDR through Homologous Recombination } \\
\text { (HRR) or Single Strand Annealing (SSA) }\end{array}$ & 19 & $5.88 \mathrm{E}-12$ \\
\hline R-HSA-73933 & Resolution of Abasic Sites (AP sites) & 13 & $1.53 \mathrm{E}-11$ \\
\hline R-HSA-176187 & $\begin{array}{l}\text { Activation of ATR in response to replication } \\
\text { stress }\end{array}$ & 12 & $2.21 \mathrm{E}-11$ \\
\hline $\begin{array}{l}\text { R-HSA- } \\
4615885\end{array}$ & SUMOylation of DNA replication proteins & 13 & $2.52 \mathrm{E}-11$ \\
\hline
\end{tabular}




\section{Table 4(on next page)}

Calculation results of 9 algorithms.

The row corresponds to gene, and the column corresponds to algorithm. Each value represents a score. 


\begin{tabular}{|c|c|c|c|c|c|c|c|c|c|}
\hline $\begin{array}{l}\text { Hub } \\
\text { gene }\end{array}$ & DMNC & MNC & Degree & EPC & BottleNeck & Closeness & Radiality & Betweenness & Stress \\
\hline NUSAP1 & 1.0893 & 51 & 51 & 19.988 & 9 & 101.33333 & 5.09091 & 96.90787 & 1902 \\
\hline MCM3 & 0.91716 & 69 & 69 & 22.824 & 23 & 111.5 & 5.24242 & 372.309 & 7082 \\
\hline TRMT6 & 0.47733 & 9 & 11 & 2.093 & 6 & 69.78333 & 4.4 & 1264.09432 & 10640 \\
\hline RFC3 & 0.88611 & 60 & 60 & 20.772 & 10 & 107.25 & 5.19394 & 542.48026 & 7874 \\
\hline POLA2 & 1.16211 & 36 & 36 & 16.597 & 1 & 93.33333 & 4.97576 & 27.46845 & 786 \\
\hline AURKA & 0.83591 & 71 & 71 & 23.302 & 7 & 112.5 & 5.25455 & 944.81247 & 9646 \\
\hline $\mathrm{CDC} 20$ & 0.75746 & 80 & 80 & 23.462 & 5 & 117.66667 & 5.33939 & 1556.30666 & 16426 \\
\hline TOP2A & 0.81632 & 76 & 76 & 23.363 & 2 & 115.66667 & 5.30909 & 1108.53404 & 13048 \\
\hline ECT2 & 1.16516 & 30 & 30 & 14.084 & 1 & 89.33333 & 4.90303 & 9.41338 & 276 \\
\hline TK1 & 1.14366 & 41 & 41 & 17.127 & 1 & 95.75 & 5 & 60.29409 & 1056 \\
\hline MCM2 & 0.93961 & 69 & 69 & 22.807 & 5 & 111.58333 & 5.25455 & 827.49161 & 8772 \\
\hline FEN1 & 0.82386 & 73 & 73 & 22.298 & 4 & 114.25 & 5.29697 & 1278.36825 & 12488 \\
\hline NOP58 & 0.2704 & 15 & 16 & 2.45 & 10 & 76.91667 & 4.61818 & 2059.91502 & 14078 \\
\hline GINS2 & 1.15247 & 50 & 50 & 19.731 & 1 & 101 & 5.09091 & 105.79312 & 1852 \\
\hline NCAPD2 & 1.19618 & 38 & 39 & 17.413 & 7 & 96.25 & 5.05455 & 1216.19414 & 12076 \\
\hline KPNA2 & 0.97174 & 51 & 51 & 19.066 & 8 & 104.41667 & 5.21212 & 1504.41719 & 14138 \\
\hline
\end{tabular}




\section{Table 5 (on next page)}

Methylation of hub genes.

The $P$-value was obtained by $t$-test. Methylation status was obtained by analyzing GSE73003, and expression status was obtained by analyzing TCGA data set. 


\begin{tabular}{ccccc}
\hline Hub gene & $\begin{array}{c}\text { Methylation } \\
\text { status }\end{array}$ & $\boldsymbol{P}$-value & $\begin{array}{c}\text { Expression } \\
\text { status }\end{array}$ & $\boldsymbol{P}$-value \\
\hline CDC20 & Hypomethylation & $8.94 \mathrm{E}-05$ & High expression & $2.11 \mathrm{E}-32$ \\
TOP2A & Hypomethylation & $4.41 \mathrm{E}-02$ & High expression & $2.70 \mathrm{E}-41$ \\
TK1 & Hypomethylation & $4.78 \mathrm{E}-04$ & High expression & $1.02 \mathrm{E}-49$ \\
FEN1 & Hypomethylation & $4.06 \mathrm{E}-02$ & High expression & $8.23 \mathrm{E}-62$ \\
\hline
\end{tabular}

1

2

3

4 